J3eA - Vol. 1 - 5 (2002).

DOI : 10.1051/bib-j3ea:2002005

\title{
Introduction à la microélectronique : un TP de physique du composant
}

\author{
S. Galdin-Retailleau *, A. Bournel ** et P. Hesto *** \\ Mis en ligne le 30 mai 2002.
}

\begin{abstract}
Résumé
Nous présentons un TP de physique du composant, basé sur l'utilisation d'un logiciel de simulation des transistors MOSFET à inversion. Ce logiciel comporte deux volets. Le premier, PROF, permet de visualiser facilement et rapidement les caractéristiques électriques externes des MOSFET, issues d'un modèle SPICE. Le deuxième, MCARLO, offre la possibilité d'étudier quelques paramètres microscopiques caractérisant le transport des porteurs de charge dans le canal du transistor (vitesse, énergie, trajectoire), calculés à l'aide de modèles physiques simplifiés (« énergie-balance » et simulation particulaire Monte Carlo). Cette séance de TP aide à la compréhension des phénomènes physiques mis en jeu dans les MOSFET, et met en valeur les problèmes posés par ces phénomènes pour le dimensionnement des composants au sein des circuits logiques CMOS.

Mots-clés. microélectronique, MOSFET, transport, caractérisation, SPICE, Monte Carlo, dérive, diffusion.
\end{abstract}

(C) EDP Sciences, 2002.

Niveau de connaissances requis. Cours classique de physique du composant : fonctionnement d'un MOSFET normally off.

Niveau des étudiants. Deuxième cycle, licence EEA.

* Sylvie Galdin-Retailleau, ancienne élève de l'ENS de Cachan, a été reçue à l'agrégation de physique appliquée en 1988 et a obtenu le doctorat en sciences de l'université Paris-Sud en 1992. Depuis octobre 1992, elle enseigne dans les domaines de la physique des composants, de l'électronique analogique et numérique au niveau DEUG, licence, maîtrise et DEA à l'université Paris-Sud.

Ses thèmes de recherche développés à l'Institut d'\&Eacutelectronique Fondamentale d'Orsay sont centrés sur l'étude théorique de dispositifs pour la microélectronique avancée allant du transport à la porte élémentaire. Elle a notamment étudié les dispositifs à hétérojonctions IV-IV en technologie bipolaire et à effet de champ. Ses préoccupations portent désormais sur la modélisation des composants à effet de champ ultimes et des dispositifs semi-conducteurs à effets quantiques. Elle occupe actuellement un poste de professeur à l'université Paris XI et est responsable de la maîtrise EEA (Électronique, Électrotechnique, Automatique).

Adresse postale : Institut d'Électronique fondamentale (IEF), bâtiment 220, Université Paris Sud, 91405 Orsay Cedex, France.

email : sylvie.retailleau@ief.u-psud.fr

** Arnaud Bournel est un ancien élève de l'ENS de Cachan. Il a été reçu à l'agrégation de physique appliquée en 1995 et a obtenu le doctorat en sciences de l'Université Paris-Sud en janvier 1999. Depuis octobre 1999, il occupe un poste de maître de conférences à l'Université Paris-Sud, et effectue son activité de recherche à l'Institut d'Électronique Fondamentale d'Orsay au sein de l'opération « Magnétoélectronique dans les systèmes mésoscopiques ». 
Son activité de recherche actuelle concerne les développements de l'électronique de spin dans les structures à semiconducteurs. L'ensemble de ses travaux de recherche a de plus été reconnu par le prix "Jeune Chercheur" du Club Nanotechnologie qu'il a obtenu en novembre 2000, et par des invitations à des communications orales sur l'électronique de spin dans les semiconducteurs. Ses activités d'enseignement concernent les sciences et techniques liées aux télécommunications, essentiellement en Maîtrise EEA et dans le DESS « Systèmes électroniques » d'Orsay.

Adresse postale : Institut d'Électronique fondamentale (IEF), bâtiment 220, Université Paris Sud, 91405 Orsay Cedex, France.

*** Patrice Hesto est un ancien élève de l'ENS de Cachan. Il a passé son doctorat d'État à l'Université Paris XI en 1984, sur l'étude théorique par simulation Monte Carlo du transport balistique dans les composés III-V. En 1984, il a rejoint le Laboratoire de Microstructures et Microélectronique (L2M) de Bagneux, dans lequel il a pris la responsabilité d'un groupe de recherche sur les transistors bipolaires à hétérojonction III-V. Depuis 1987, il développe ses activités de recherche à l'Institut d'Électronique Fondamentale de Paris XI. Il a travaillé notamment dans les domaines des systèmes micro-électromécaniques et des transistors à base de couches minces organiques. Ses thèmes de recherche portent désormais sur la magnétoélectronique dans les structures à semiconducteurs III-V et sur l'étude théorique du transport dans les transistors fortement submicroniques. Il occupe actuellement un poste de professeur à l'Université Paris XI et est le responsable du Pôle de Microélectronique Paris-Sud (PMIPS) du Centre National de Formation en Microélectronique (CNFM).

Adresse postale : Institut d'Électronique fondamentale (IEF), bâtiment 220, Université Paris Sud, 91405 Orsay Cedex, France.

\section{Introduction}

L'enseignement de la physique du composant souffre souvent d'une présentation trop théorique basée sur des équations plus ou moins complexes décrivant les propriétés des semiconducteurs et les phénomènes de transport. Il est alors perçu par les étudiants comme un enseignement rébarbatif, d'où leur peu de motivation. Il est certain que la mise en place de travaux pratiques dans ce type d'enseignement peut aider à la compréhension des phénomènes physiques en concrétisant certains effets et en rendant les étudiants plus actifs.

Nous présentons dans cet article un TP de physique du composant de 4 heures sur le transistor MOSFET " normally off », destiné à des étudiants de licence EEA. Ce TP utilise deux modules d'un logiciel développé à l'INSA de Toulouse [1] en collaboration, pour le module MCARLO [2], avec l'IEF d'Orsay :

- Le module PROF : son utilisation permet, d'une part, de mieux comprendre l'allure des caractéristiques $I(V)$ du transistor et, d'autre part, d'étudier l'influence de certains paramètres (longueur $L$ et largeur $W$ de la grille, température $T$...) sur ces caractéristiques.

- Le module MCARLO : son utilisation permet de visualiser l'évolution le long du canal du transistor de l'énergie totale des électrons, de leur énergie potentielle, de leur vitesse et de leur température, ainsi que la trajectoire des porteurs dans le canal, suivant les paramètres du transistor $\left(V_{G S}, V_{D S}, L, W \ldots\right)$.

Ce logiciel fonctionne sous DOS sur PC.

\section{PROF}

\subsection{Présentation du logiciel}

PROF est un outil interactif permettant de tracer les caractéristiques électriques de différents composants : diodes, transistors bipolaires et MOS. Ces caractéristiques sont tracées en tenant compte des effets de la température, de la tension d'alimentation, des dimensions géométriques du dispositif. 
Pour le transistor MOS, ce module utilise pour simuler ces caractéristiques un modèle basé sur le simulateur SPICE niveau 3.

L'écran est divisé en quatre parties, comme illustré sur la figure 1, avec :

1. la vue en coupe du transistor MOS (en haut à gauche) ;

2. la vue de dessus du transistor MOS (en haut à droite + animation associée) ;

3. le panneau de contrôle des paramètres, $V_{G S}, V_{D S}$ et la température, ainsi qu'une indication sur l'état de polarisation du transistor (saturé, linaire, sous le seuil ou bloqué) et la valeur du courant de drain qui le traverse (en bas à gauche) ;

4. les caractéristiques courant/tension du transistor MOS (en bas à droite), le point de polarisation étant repéré par l'intersection de deux lignes horizontale et verticale.

Vue en coupe du transistor MOS

Vue du dessus du transistor MOS (Voir animation 1)

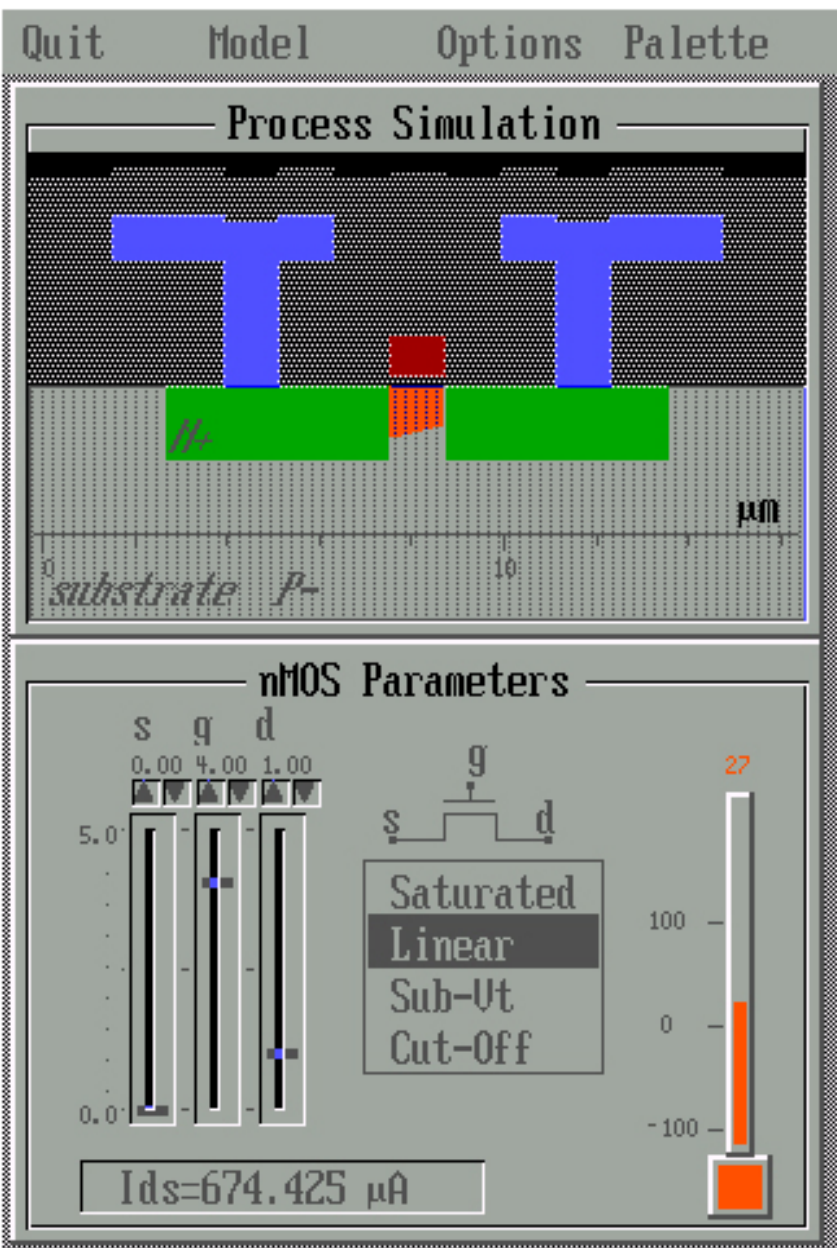

Panneau de contrôle des parametres, $V G S, V D S$ et la température,ainsi qu'une indication sur l'état de polarisation du transistor (saturé, linéaire, sous le seuil ou bloqué) et la valeur du courant de drain qui le traverse.
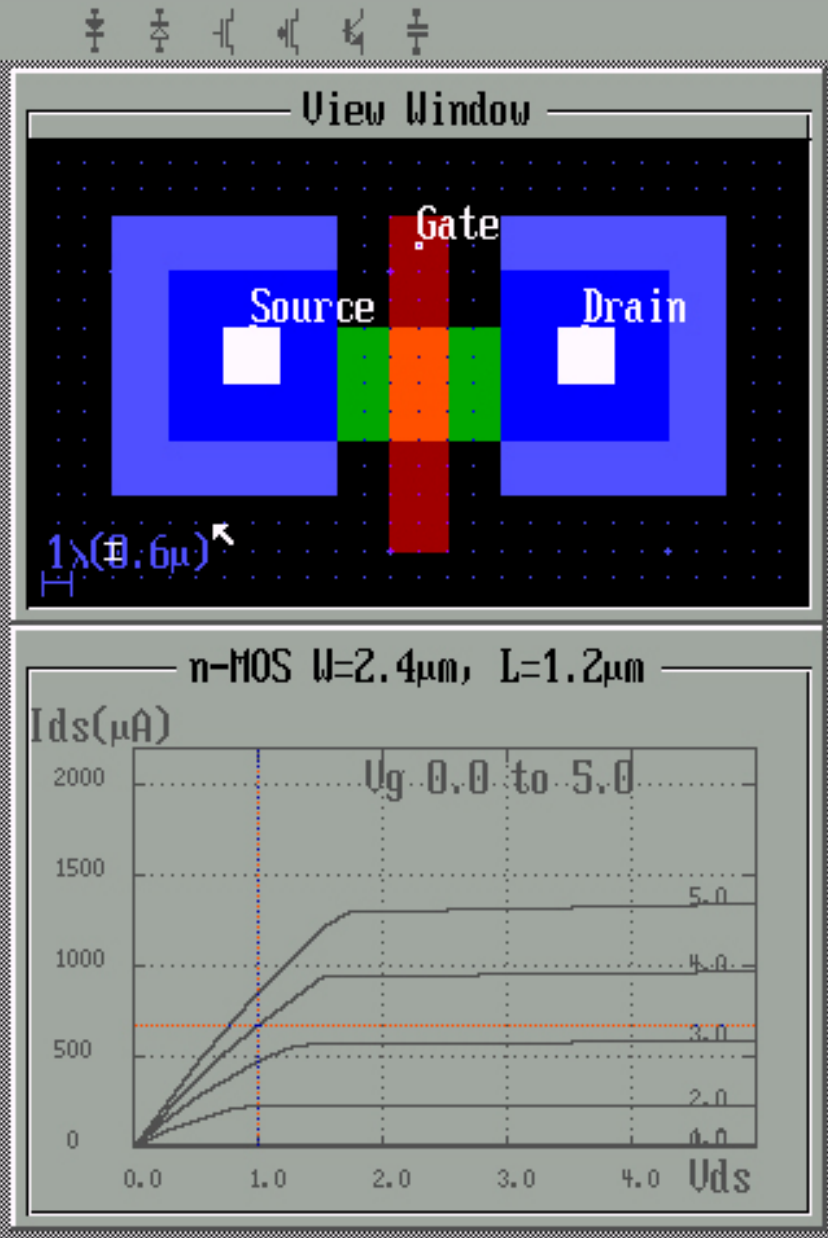

Caratéristiques courant/tension du transistor MOS, le point de polarisation étant repéré par l'intersection des deux lignes horizontale et verticale.

Figure 1. Écran standard de PROF. 
a)

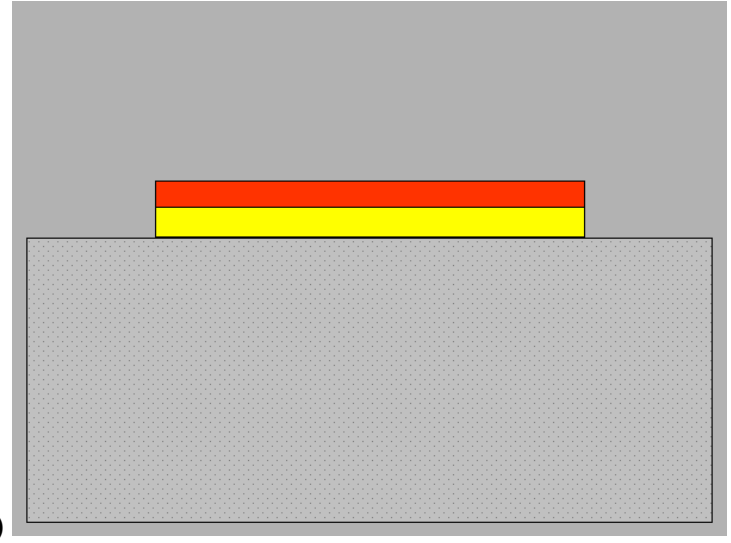

c)

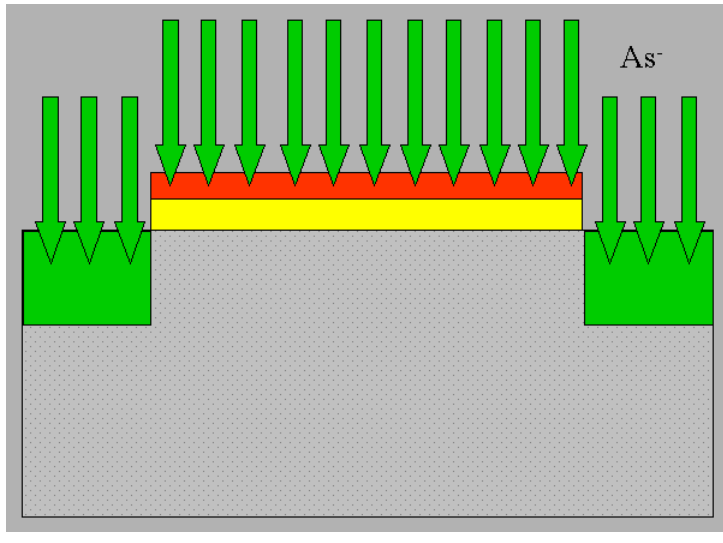

e)

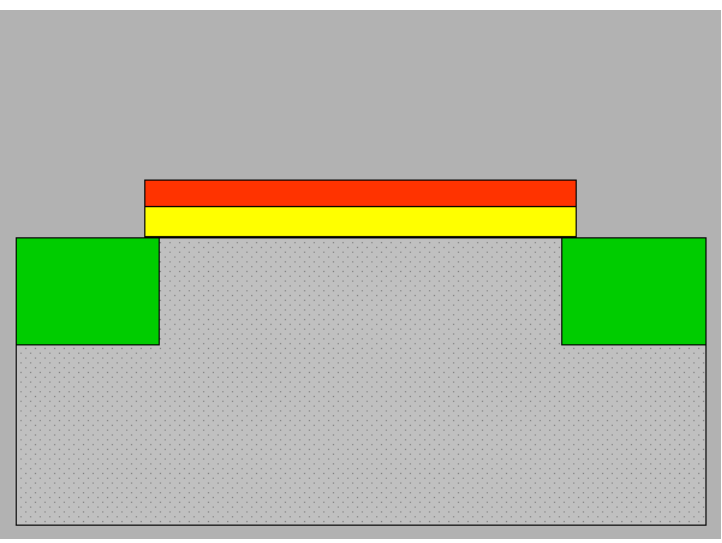

b)

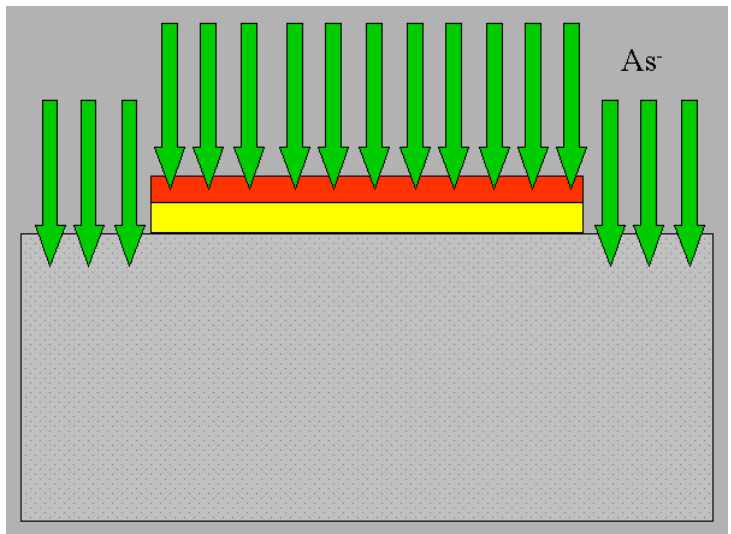

d)

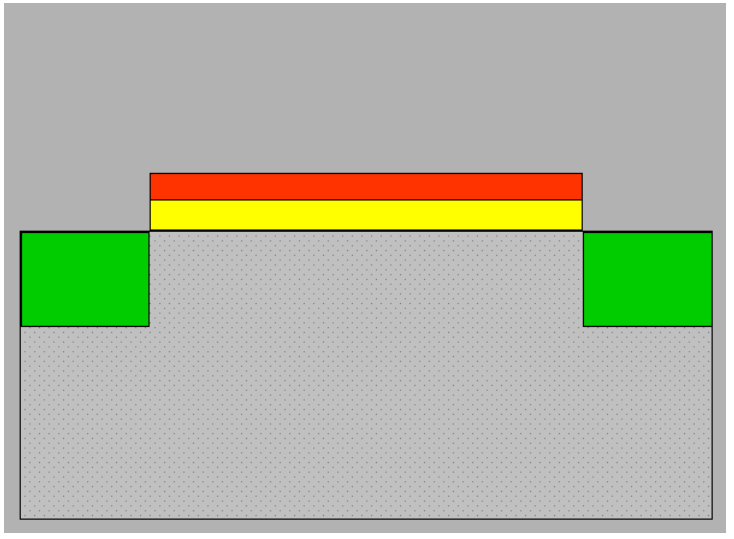

f)

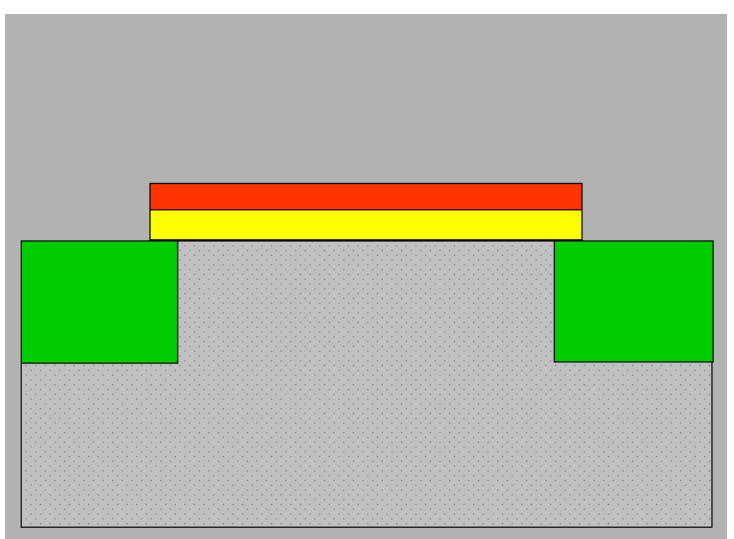

Animation 1 : Implantation des caissons source et drain - a)

b) Implantation source et drain à travers le masque de grille.

c) Les ions pénètrent dans le Si là où il n'y a pas de masque.

d) Début du recuit post-implantation.

e) Les impuretés implantées diffusent au cours du recuit post-implantation.

f) Début du recuit post-implantation. 


\subsection{Présentation du transistor MOSFET}

Un MOSFET normally off (à enrichissement) à canal $N$ est constitué de deux zones très dopées de type $N$ (la source et le drain) réalisées dans un substrat de type $P$ sur lequel on a déposé une couche d'oxyde et une électrode de grille (Fig. 2).

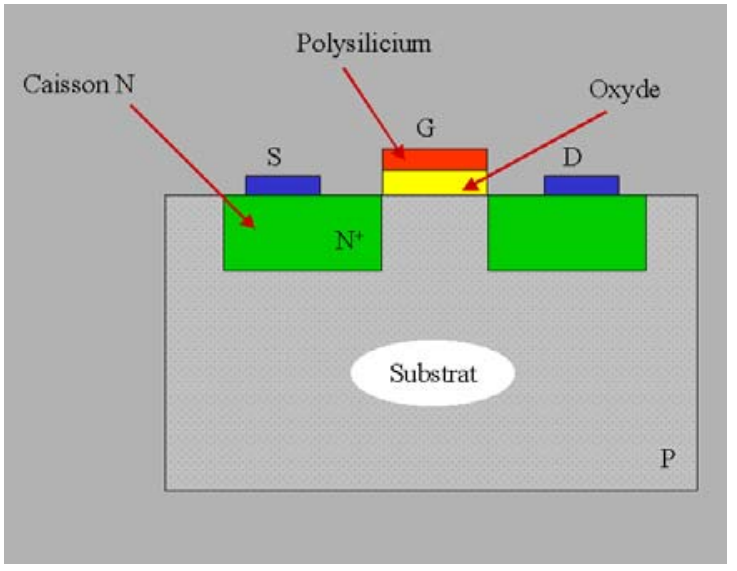

b)
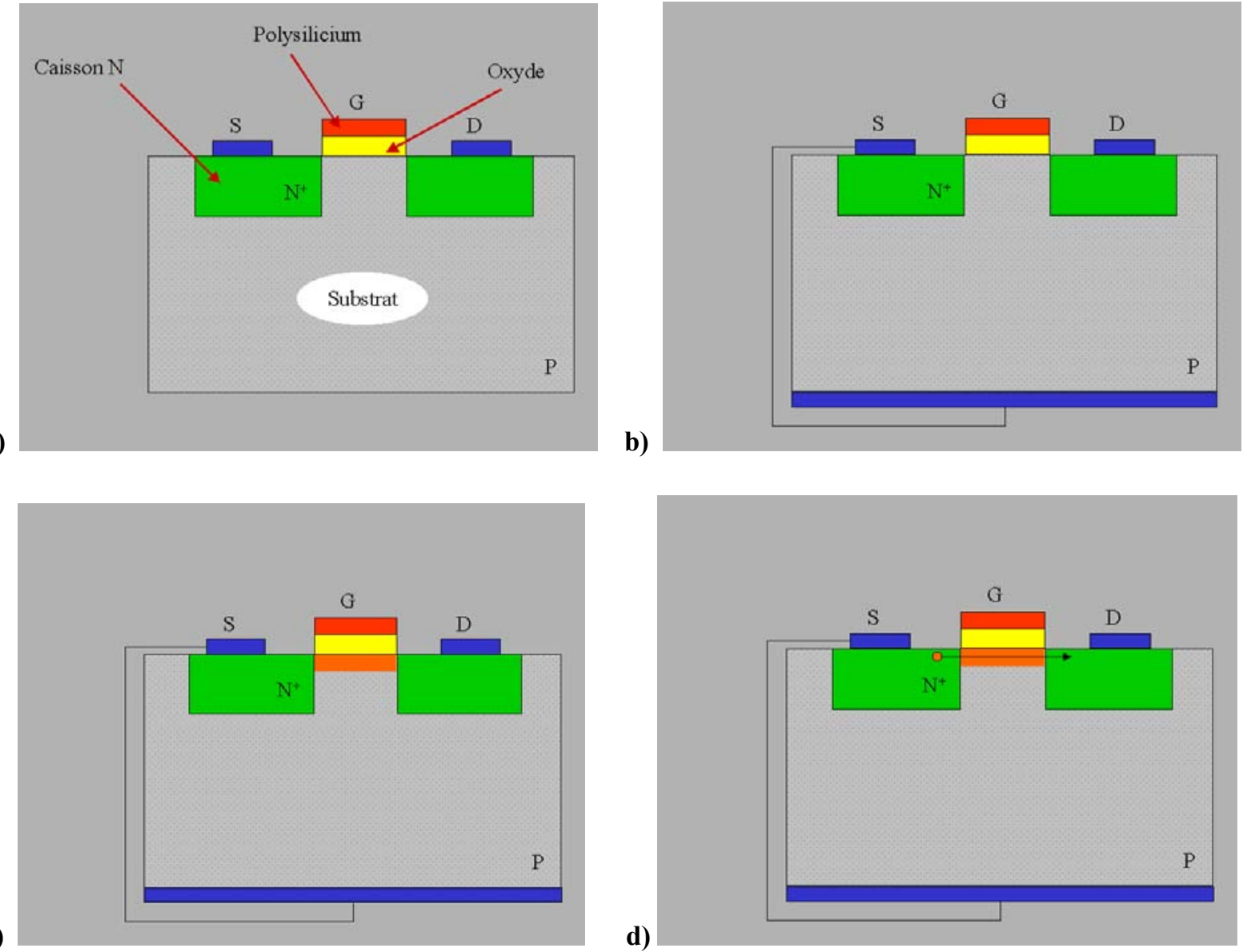

Figure 2. Géométrie du NMOS à inversion - a)

b) Polarisation du substrat.

c) Formation d'un canal d'inversion.

d) Passage des porteurs de la source au drain.

Dans la plupart des cas le substrat (B) est au même potentiel que la source, pris comme potentiel de référence, afin d'éviter les fuites de courant par le substrat (Fig. 2). Pour faire passer un flux d'électrons de la source au drain, il faut réaliser deux conditions (Fig. 2) :

i. présence des électrons entre la source et le drain : formation d'un canal d'inversion

ii. mouvement des électrons de la source vers le drain. 


\subsection{Formation du canal}

Afin d'attirer les électrons sous la grille grâce à la structure capacitive MOS, il faut imposer une polarisation positive de la grille. Mais, le premier effet d'appliquer $V_{G S}>0 \mathrm{~V}$ est de repousser les trous initialement présents sous la grille vers le fond du substrat et de créer une zone de charge d'espace $(\mathrm{ZCE})$ à l'interface $\mathrm{Si}-\mathrm{P} / \mathrm{SiO}{ }_{2}(\mathrm{Fig}$. $3)$.

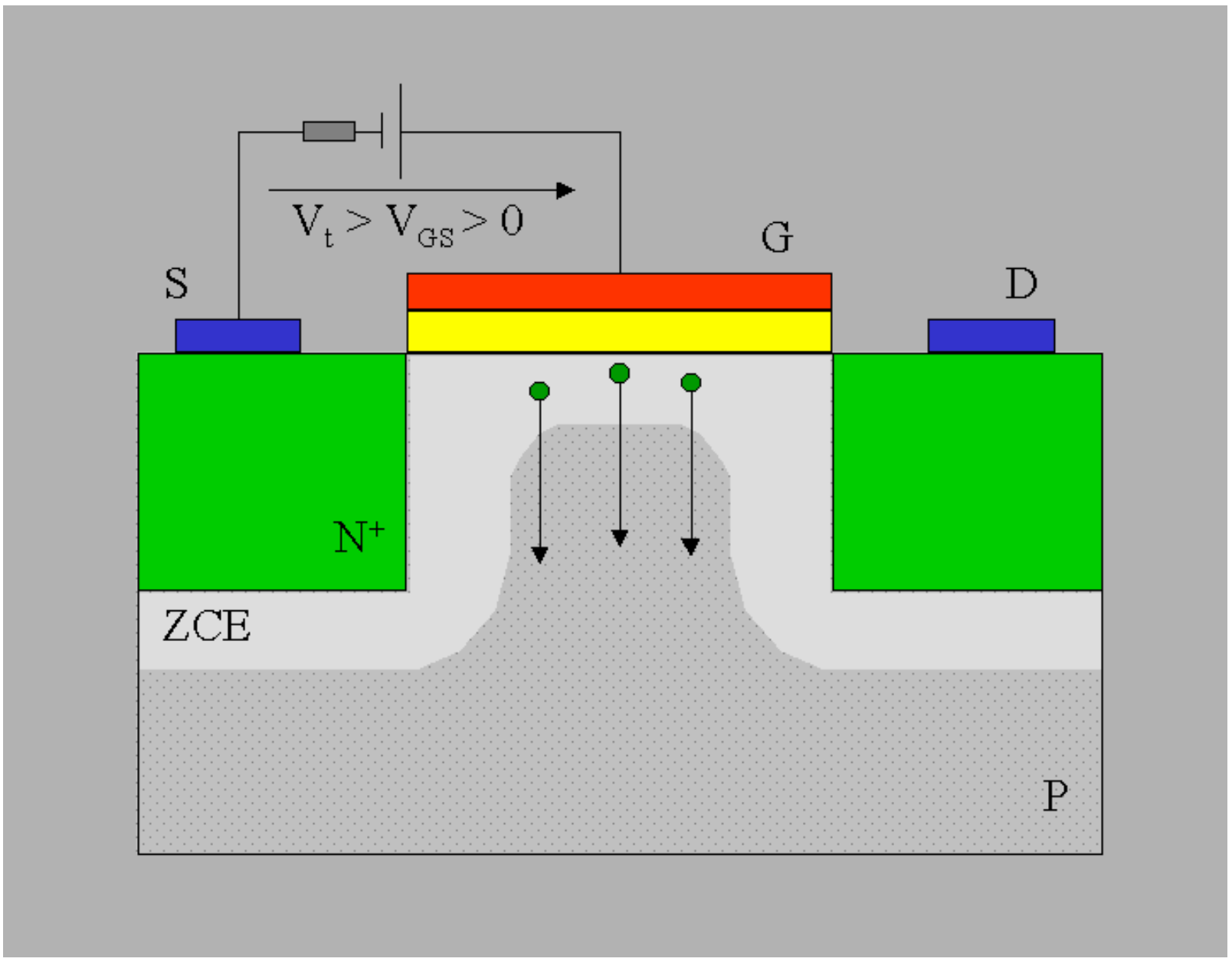

Figure 3. Désertion en trous sous la grille.

C'est seulement pour une certaine tension $V_{G S}=V_{t}, V_{t}$ appelée tension de seuil d'inversion, qu'apparaît la couche d'inversion. La valeur de $V_{t}$ peut être ajustée en jouant sur le dopage du substrat et sur le matériau de grille.

Les figure 4 et 5 (haut et bas) sont des copies d'écran des fenêtres 1 et 3 du module PROF (Fig. 1) qui illustrent l'état $V_{G S}<V_{t}$, appelé régime sous le seuil. 

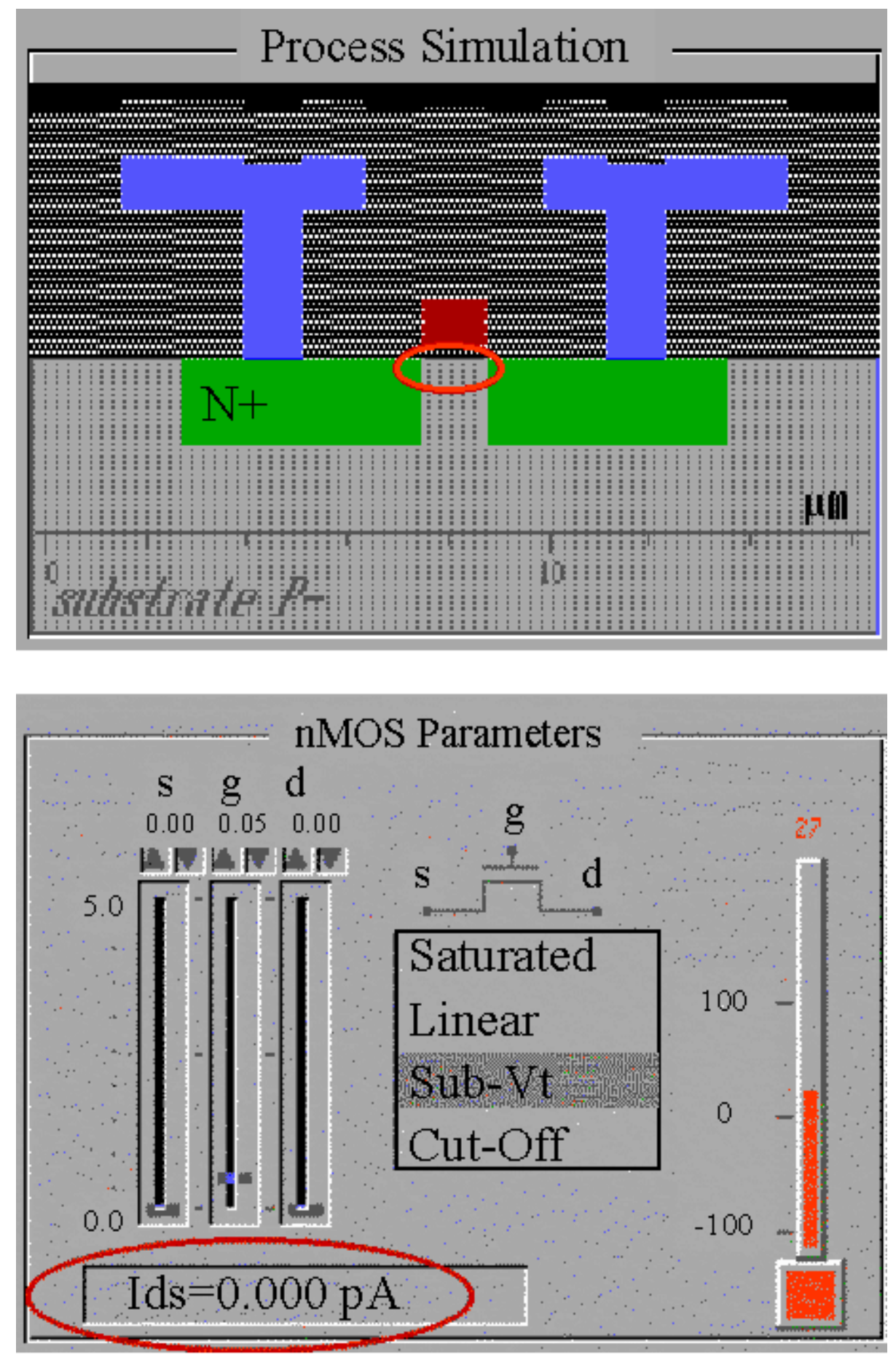

Fig. 4 et 5 : Régime sous le seuil.

Fig. 4 (en haut) : Absence de canal en régime sous le seuil.

Fig. 5 (en bas) : Absence de courant en régime sous le seuil.

$\mathrm{Si}$ on applique sur la grille une tension supérieure à la tension de seuil $V_{t}$, on induit une couche d'inversion à l'interface $\mathrm{Si}-\mathrm{P} / \mathrm{SiO}_{2}$. La couche d'inversion n'est plus créée par génération dans la ZCE, comme dans le cas d'une capacité MOS, mais par diffusion des électrons depuis les réservoirs d'électrons source et drain (Fig. 6). Ce phénomène est donc très rapide. 

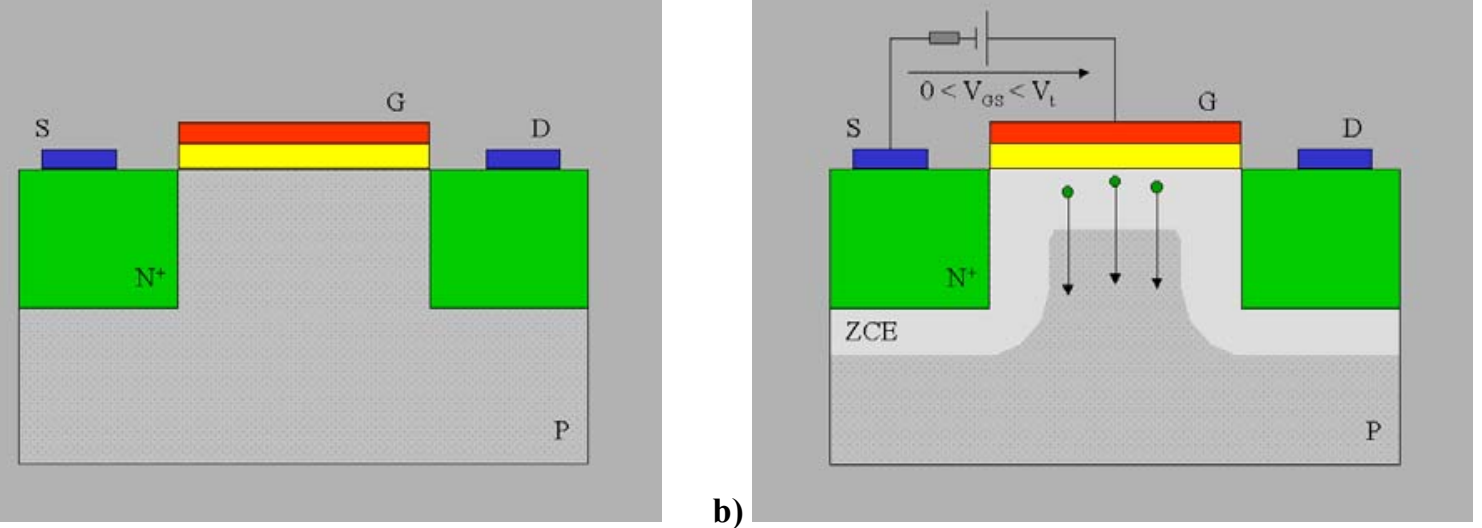

a)

b)
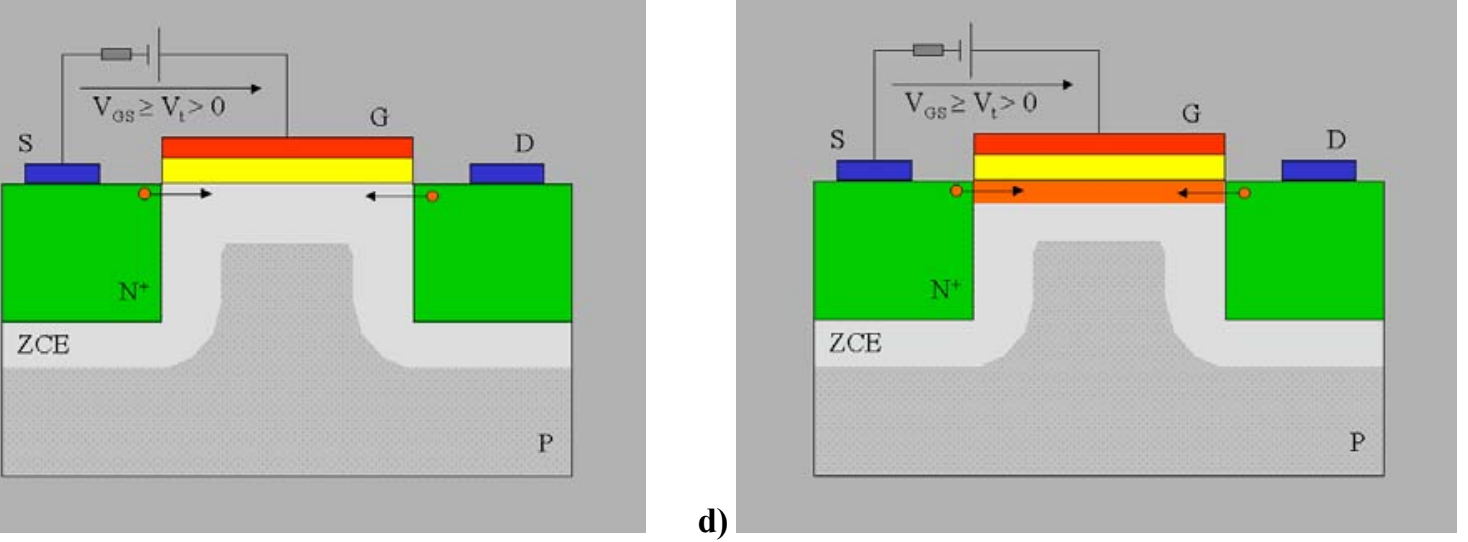

Figure 6 : Formation du canal par diffusion depuis les caissons source-drain. - a)

b) Pour $V_{G S}$ compris entre 0 et $V_{t}$, une ZCE se forme sous la grille.

c) Quand $V_{G S}$ s'approche de $V_{t}$, les électrons des réservoirs de source et drain sont attirés par la grille en nombre de plus en plus grand...

d) ...ainsi le canal est complètement constitué pour $V_{G S}$ supérieur à $V_{t}$. 
La création de la couche d'inversion $\left(V_{G S}>V_{t}\right)$ assure la condition (i) énoncée précédemment, mais elle est cependant insuffisante à elle seule pour conduire au passage d'un courant ( $I_{D}=0 \mathrm{~A}$, Fig. 7).

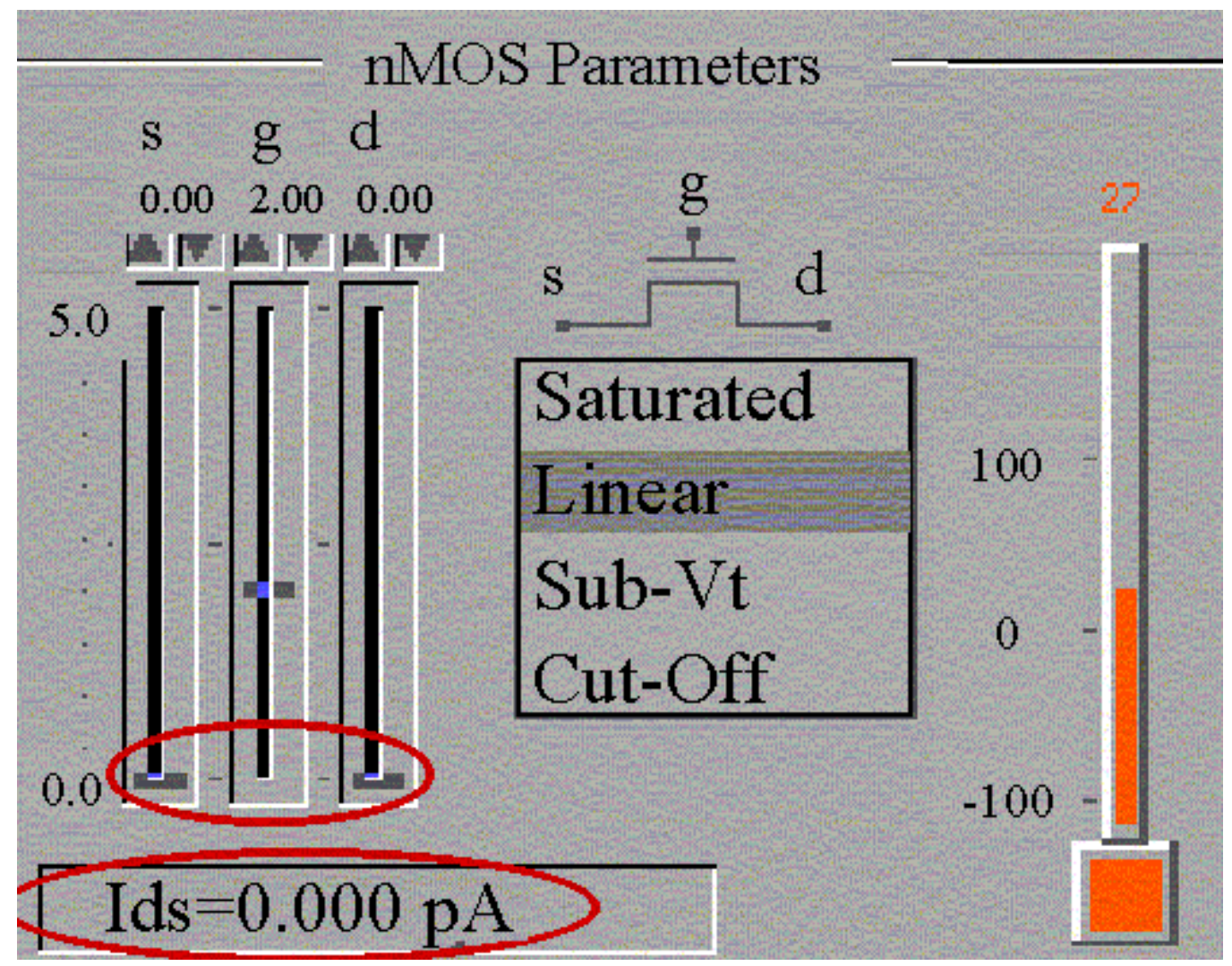

Fig. 7 : Le canal ne suffit pas...

Annexe 1 : Illustration de la formation du canal par un autre logiciel de simulation $[3,4]$

\subsection{Régimes de fonctionnement}

\subsubsection{Régime ohmique}

Le mouvement des électrons de la source vers le drain requiert l'application d'une tension de polarisation $V_{D S}>0 \mathrm{~V}$ (Fig. 8, haut), afin d'imposer un champ électrique accélérateur dans le canal orienté du drain vers la source.

La différence de potentiel entre la grille et le canal décroît alors de la source vers le drain, ce qui conduit à une diminution de la densité surfacique d'électrons dans le canal depuis la source jusqu'au drain (Fig. 8, milieu).

Tant que la tension $V_{D S}$ reste faible, c'est-à-dire tant qu'il existe un canal d'électrons qui relie la source au drain, le canal induit se comporte comme une simple résistance : on est dans la région ohmique (Fig. 8, bas).

Rappelons que le point de polarisation $\left(V_{D S} ; I_{D}\right)$ est indiqué sur le réseau de caractéristiques par l'intersection d'une droite verticale et d'une droite horizontale. 

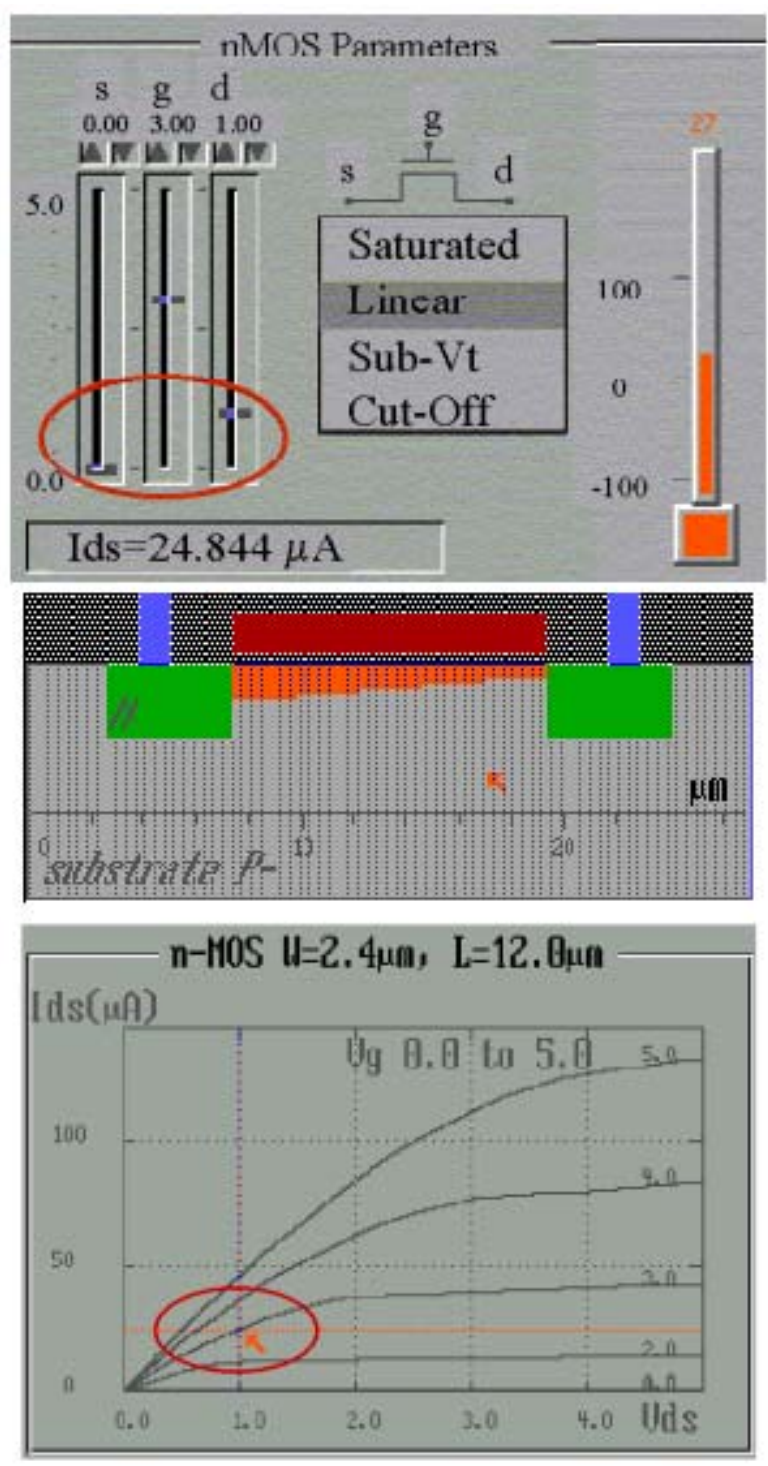

Le mouvement des électrons de la source vers le drain requiert l'application d'une tension de polarisation $V_{D S}>0 \mathrm{~V}$, afin d'imposer un champ électrique accélérateur dans le canal orienté du drain vers la source.

La différence de potentiel entre la grille et le canal décroît alors de la source vers le drain, ce qui conduit à une diminution de la densité surfacique d'électrons dans le canal depuis la source jusqu'au drain.

Tant que la tension $V_{D S}$ reste faible, c'est-à-dire tant qu'il existe un canal d'électrons qui relie la source au drain, le canal induit se comporte comme une simple résistance : on est dans la région ohmique.

\subsubsection{Régime source de courant}

Afin de décrire ce régime, il faut distinguer d'abord deux cas limites : les transistors à grille longue, soit typiquement $L$ de l'ordre de $10 \mu \mathrm{m}$, et les transistors à grille courte, soit typiquement $L$ de l'ordre de $1 \mu \mathrm{m}$.

\subsubsection{Grille longue (Fig. 9)}

Pour une tension $V_{G S}$ donnée, lorsque $V_{D S}=V_{s a t}^{L}=V_{G S}-V_{t}$, la tension $V_{G D}$ entre grille et drain est égale à la tension de seuil $V_{t}$. Par définition de la tension de seuil, il en résulte que la charge de la couche d'inversion en bout du canal est quasi nulle. On dit que le canal est pincé (voir annexe 2). La ZCE qui s'étend alors du point de pincement au caisson de drain est une zone très résistive qui supporte donc l'accroissement de tension $V_{D S}-V_{\text {sat }}^{L}$ lorsque $V_{D S}$ augmente encore.

Cette zone étant de plus très courte le champ qui y règne est intense ; il happe tous les électrons parvenus en fin de canal et les catapulte vers le drain.

Pour $V_{D S}>V_{\text {sat }}^{L}$, la tension aux bornes du canal devient donc indépendante de $V_{D S}$ et égale à $V_{\text {sat }}^{L}$. Si l'on néglige le recul du point de pincement, la quantité d'électrons dans le canal et le champ accélérateur qui y règne ne varient plus : le courant sature. 

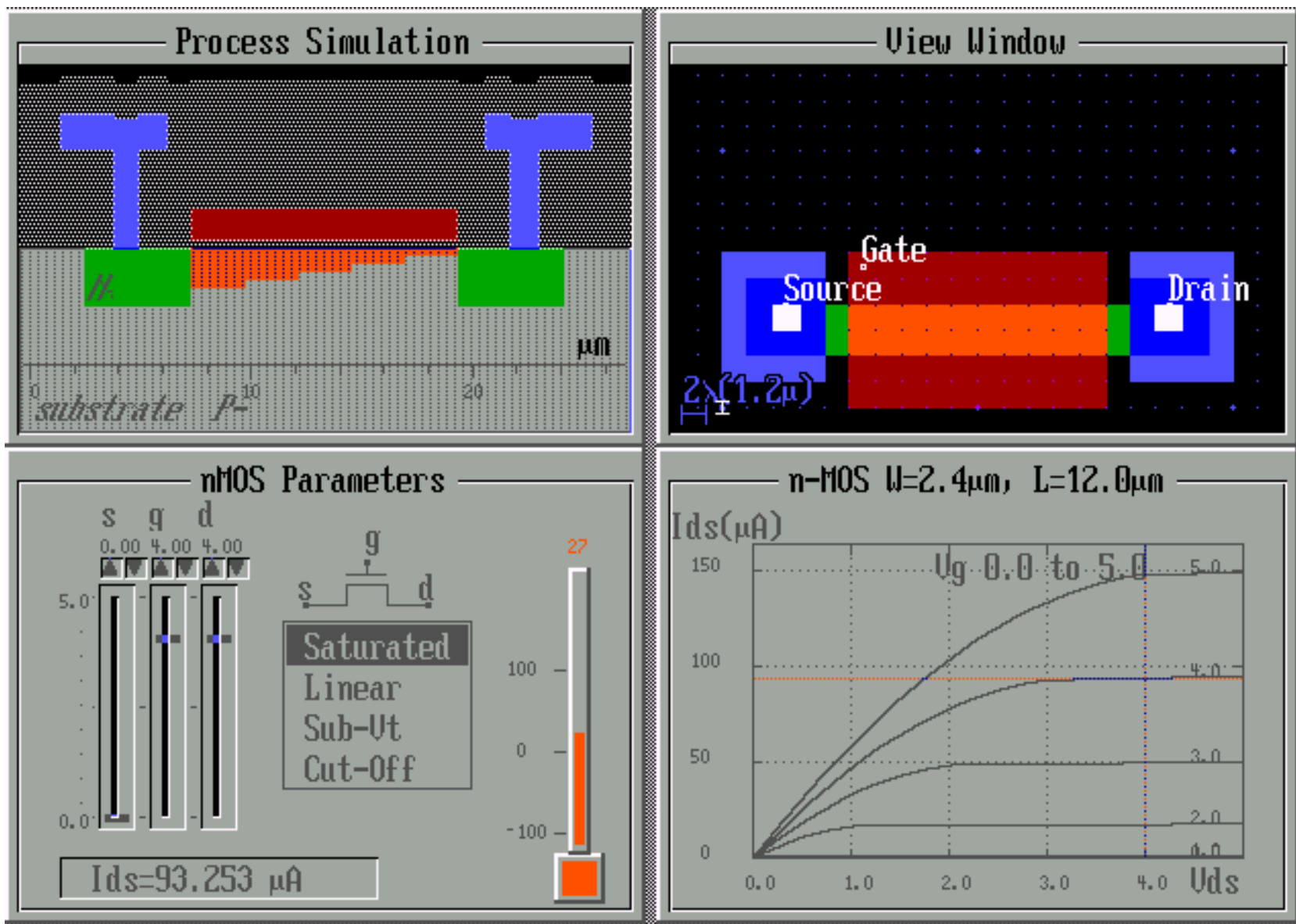

Fig. 9 : Régime source de courant et grille longue.

Annexe 2 : Pincement du canal.

\subsubsection{Grille courte (Fig. 10)}

Dans le canal d'électrons, le champ accélérateur peut s'écrire

$$
\left|E_{=}\right|=\left|\frac{V_{\mathrm{DS}}}{L}\right| .
$$

Pour une tension $V_{D S}$ donnée, le champ est d'autant plus fort que la grille du transistor est courte. Or, dans un semiconducteur, si la vitesse est bien proportionnelle au champ électrique lorsque celui-ci est faible, il n'en est pas de même à fort champ où la vitesse sature et devient constante et égale à $v_{\text {sat }}$.

Pour des grilles courtes, la saturation de la vitesse peut entraîner celle du courant dès que

$$
V_{\mathrm{DS}}=V_{\mathrm{sat}}^{\mathrm{C}}=\frac{L \nu_{\operatorname{sat}}}{\mu t}
$$

où $\mu$ est la mobilité des électrons. Dans ce cas, le phénomène de saturation par vitesse peut avoir lieu avant le pincement du canal (voir annexe 3 ). Il faut pour cela que

$$
V_{\mathrm{sat}}^{\mathrm{C}}<V_{\mathrm{zat}}^{\mathrm{I}}=V_{\mathrm{GS}}-V_{\mathrm{t}}
$$



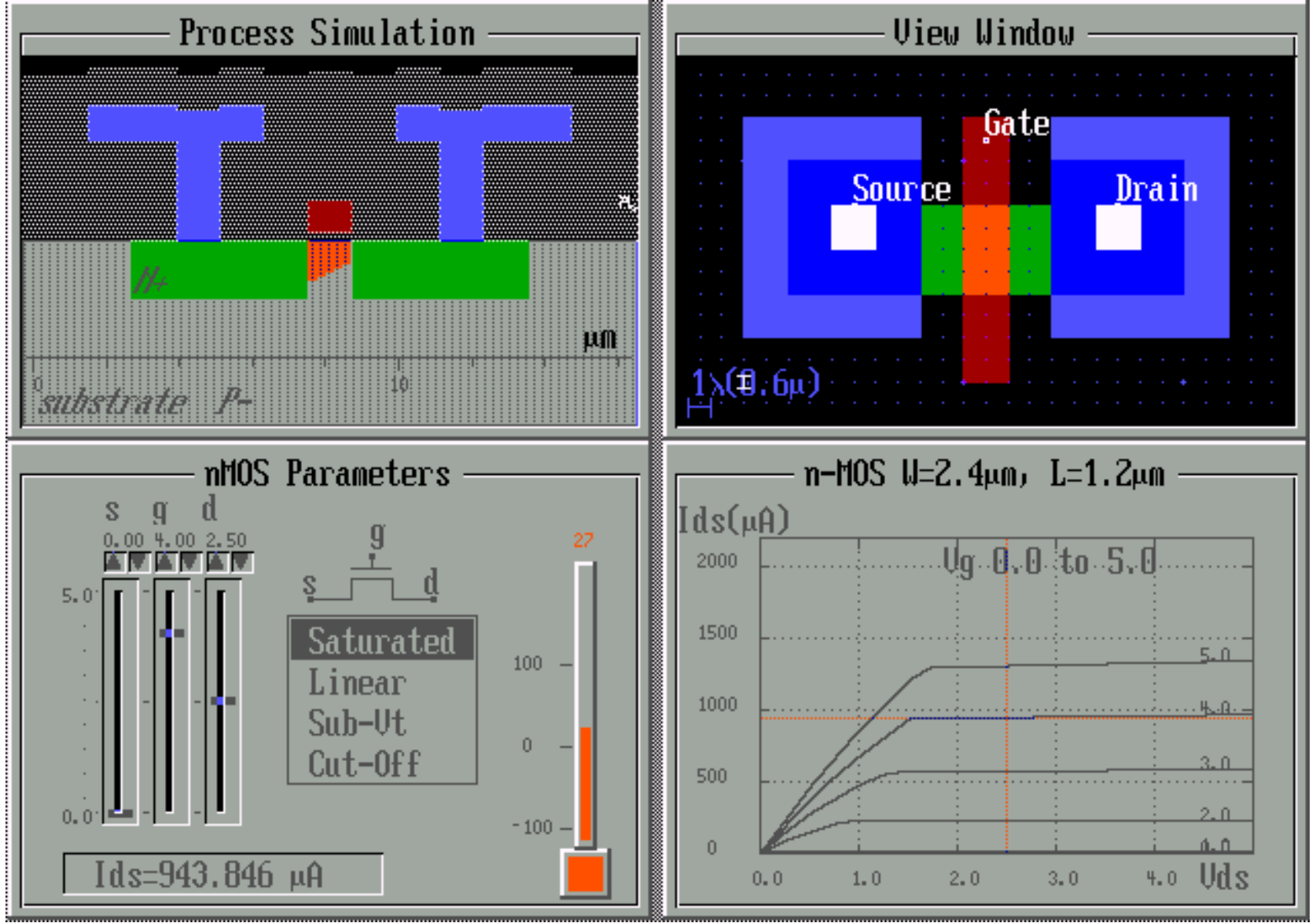

Fig. 10 : Régime source de courant et grille courte. 


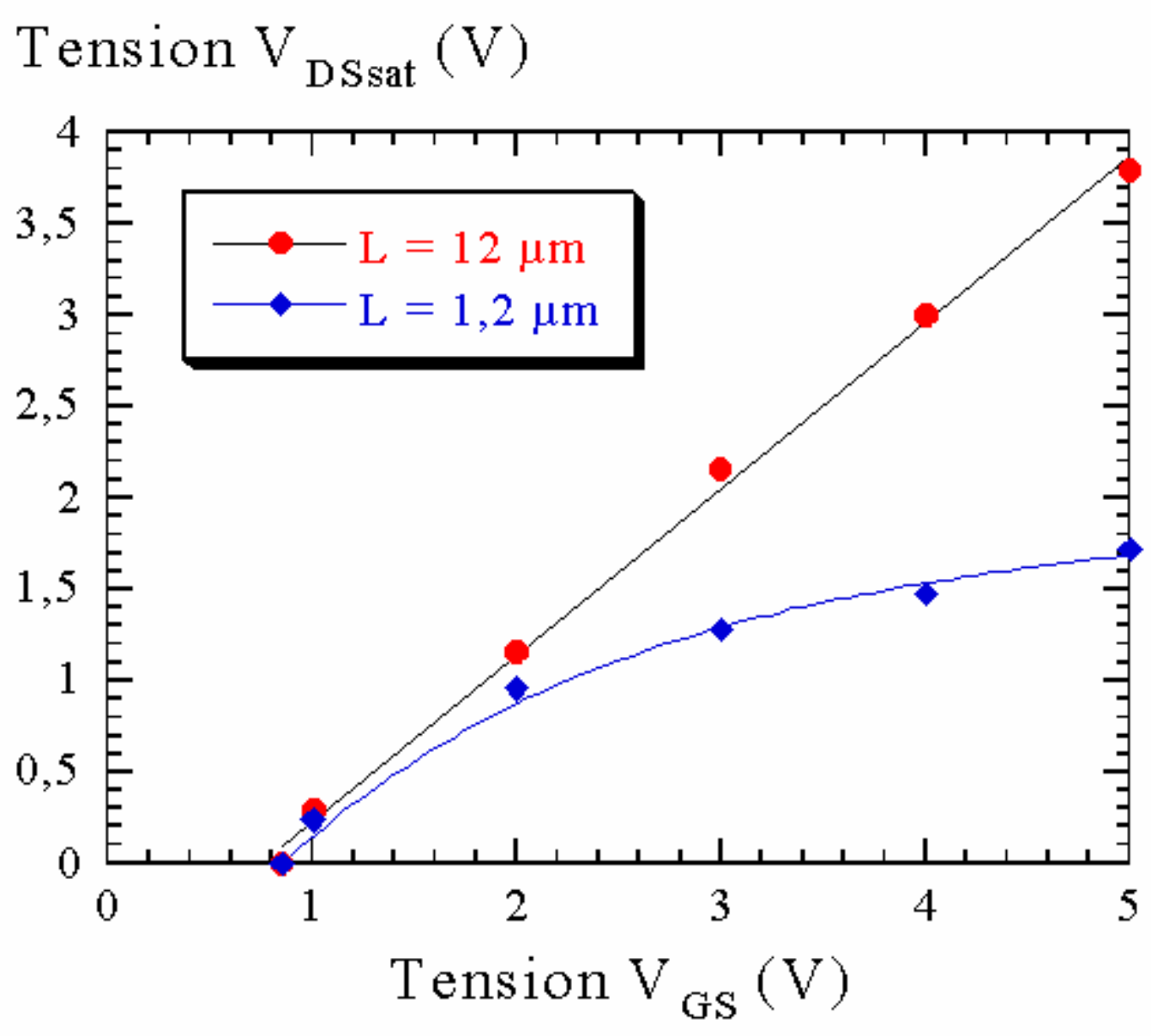

(G) EDP Sciences, 2002.

Annexe $3:$ En traçant la tension VDSsat en fonction de $V G S$ les étudiants peuvent constater que si pour un transistor à grille longue

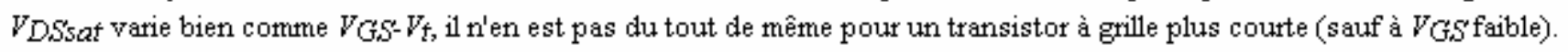

Annexe 3 : Phénomène de saturation par vitesse avant le pincement du canal.

\subsubsection{Cas général}

Remarquons que $V_{\mathrm{szz}}^{\mathrm{C}}$ est indépendante de $V_{G S}$, contrairement à ${ }^{V_{\mathrm{szt}}^{\mathrm{L}}}$. Pour un transistor de longueur de grille

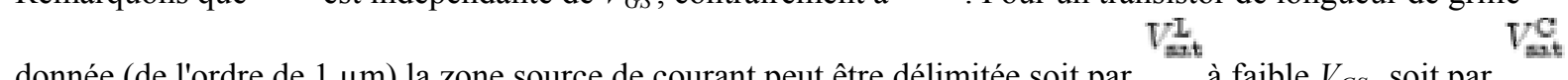
donnée (de l'ordre de $1 \mu \mathrm{m}$ ) la zone source de courant peut être délimitée soit par $\quad$ à faible $V_{G S}$, soit par à fort $V_{G S}$.

Prenons le cas d'un transistor de longueur de grille $3 \mu \mathrm{m}$ et de tension de seuil $V_{t}=1 \mathrm{~V}$ (Fig. 11) :

- $\quad$ pour $V_{G S}<3 \mathrm{~V}, V_{D S s a t}=V_{G S}-V_{t}$;

- $\quad$ pour $V_{G S}>3 \mathrm{~V}, V_{D S s a t}<V_{G S}-V_{t}$. 


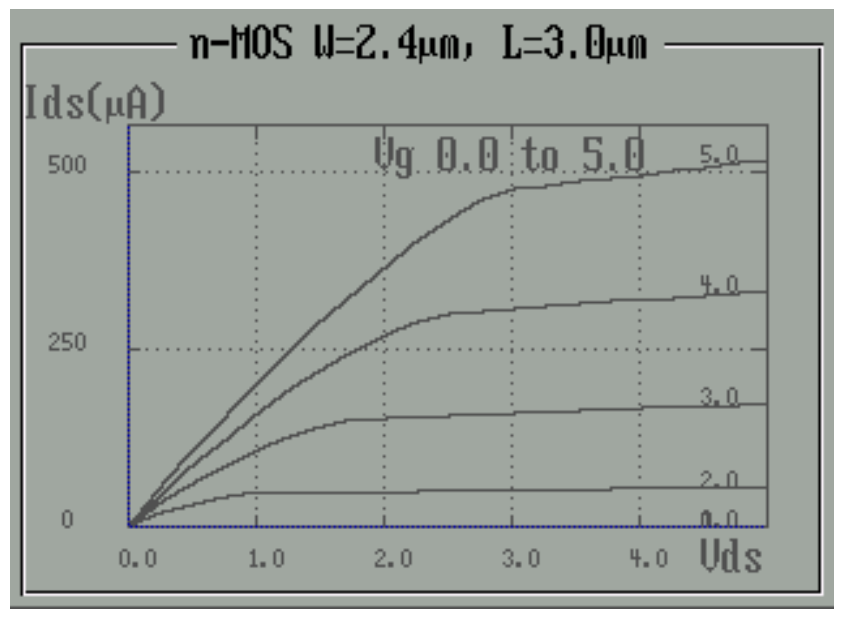

Fig. 11 : Régime source de courant et grille ni longue, ni courte.

Comment définir une tension $V_{D S s a t}$ dans le cas d'un transistor de longueur « quelconque »?

Dans le cas d'un transistor à grille « longue », on a d'après la définition de $V_{t}$ une densité surfacique d'électron $n_{s}$ nulle en fin du canal pour $V_{D S}=V_{G S}-V_{t}$. Pour avoir continuité de la densité surfacique de courant au niveau du drain $\left(j_{s}=q n_{S} v\right)$, la vitesse des porteurs en fin de canal devrait donc en toute rigueur tendre vers l'infini... La vitesse des électrons parvenus en fin de canal atteint en fait dans ce cas la valeur $v_{\text {sat }}$ au moment du « pincement » du canal.

On peut donc calculer $V_{D S s a t}$, à tension $V_{G S}$ donnée, en prenant comme critère pour le passage du régime ohmique au régime source de courant le fait que la vitesse des électrons parvenus au drain atteint $v_{\text {sat }}$. Tous calculs faits, l'utilisation de ce critère conduit à l'expression suivante de $V_{D S s a t}$ :

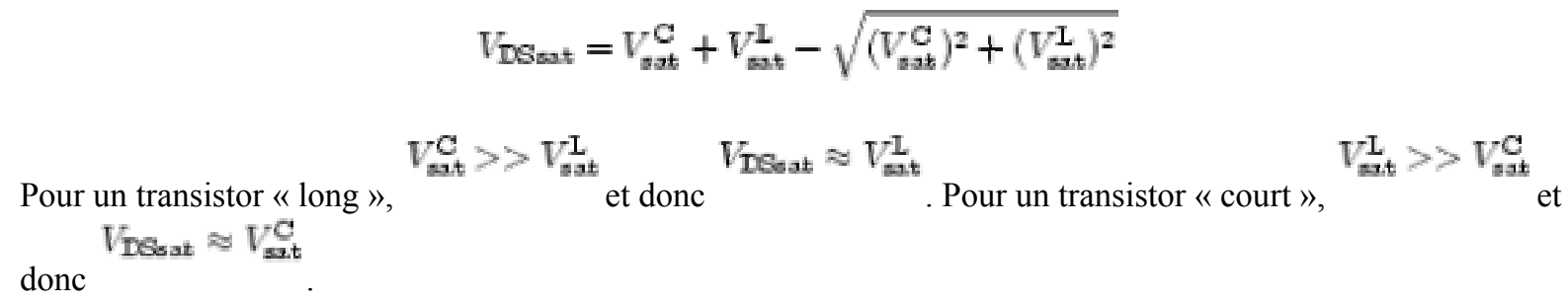

\subsubsection{Régime sous le seuil}

Jusqu'à présent, nous avons négligé le courant circulant dans le transistor pour $V_{G S}<V_{t}$. Mais bien que faible, ce courant n'est pas nul.

Le courant sous le seuil est essentiellement un courant de diffusion contrôlé par la tension de grille, d'où une variation exponentielle pour $I_{D}\left(V_{G S}\right)$.

Il est également possible de visualiser la caractéristique $\log \left(I_{D}\right)=f\left(V_{G S}\right)$ (Fig. 12). Cette visualisation permet d'étudier le comportement sous le seuil du transistor et de sensibiliser les étudiants au problème de la consommation statique des circuits CMOS qui apparaît avec l'augmentation importante de la densité d'intégration. 


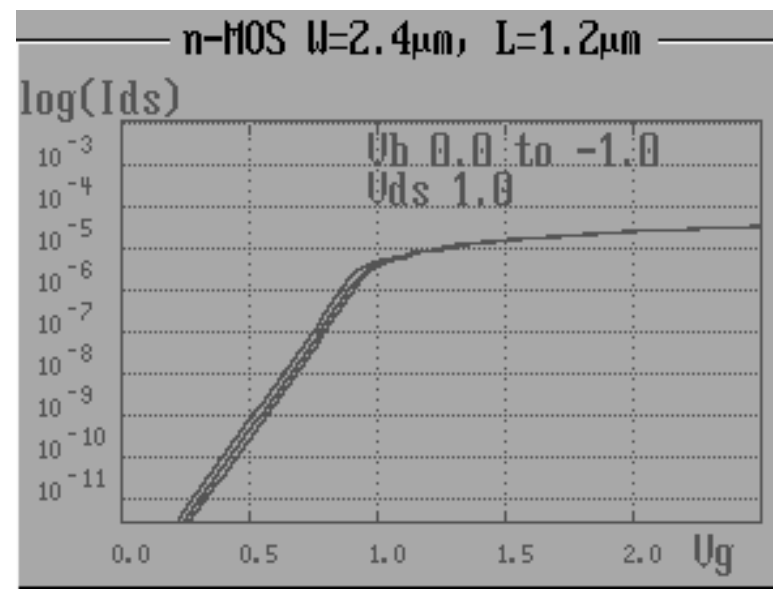

Fig. 12 : Régime sous le seuil.

On met ainsi l'accent sur le compromis à faire sur le choix de la tension de seuil par rapport aux performances des circuits CMOS :

- une amélioration de la rapidité exige de forts courants à $V_{G S}=V_{D S}=V_{D D}$, c'est-à-dire de faibles tensions de seuil ;

- alors que la diminution de la consommation statique impose de faibles courants à $V_{G S}=0 \mathrm{~V}$, c'est-à-dire des tensions de seuil élevées.

\subsection{Dimensionnement (Fig. 13)}

Le module PROF permet de modifier la longueur de la grille $L$, sa largeur $W$, la température $T$ et la tension d'alimentation $V_{D D}$.

On étudie alors l'influence de ces paramètres sur le courant de drain $I_{D}$ du transistor :

- $\quad I_{D}$ est inversement proportionnel à $L$ : le champ, et donc la vitesse, sont inversement proportionnels à $L$;

- $\quad I_{D}$ est proportionnel à $W$ : augmentation de la surface du canal ;

- $\quad I_{D}$ décroît lorsque la température augmente. Dans ce cas en effet, la mobilité, et donc la vitesse, diminuent du fait de l'augmentation des fréquences d'interaction entre les porteurs et les vibrations du réseau cristallin. 


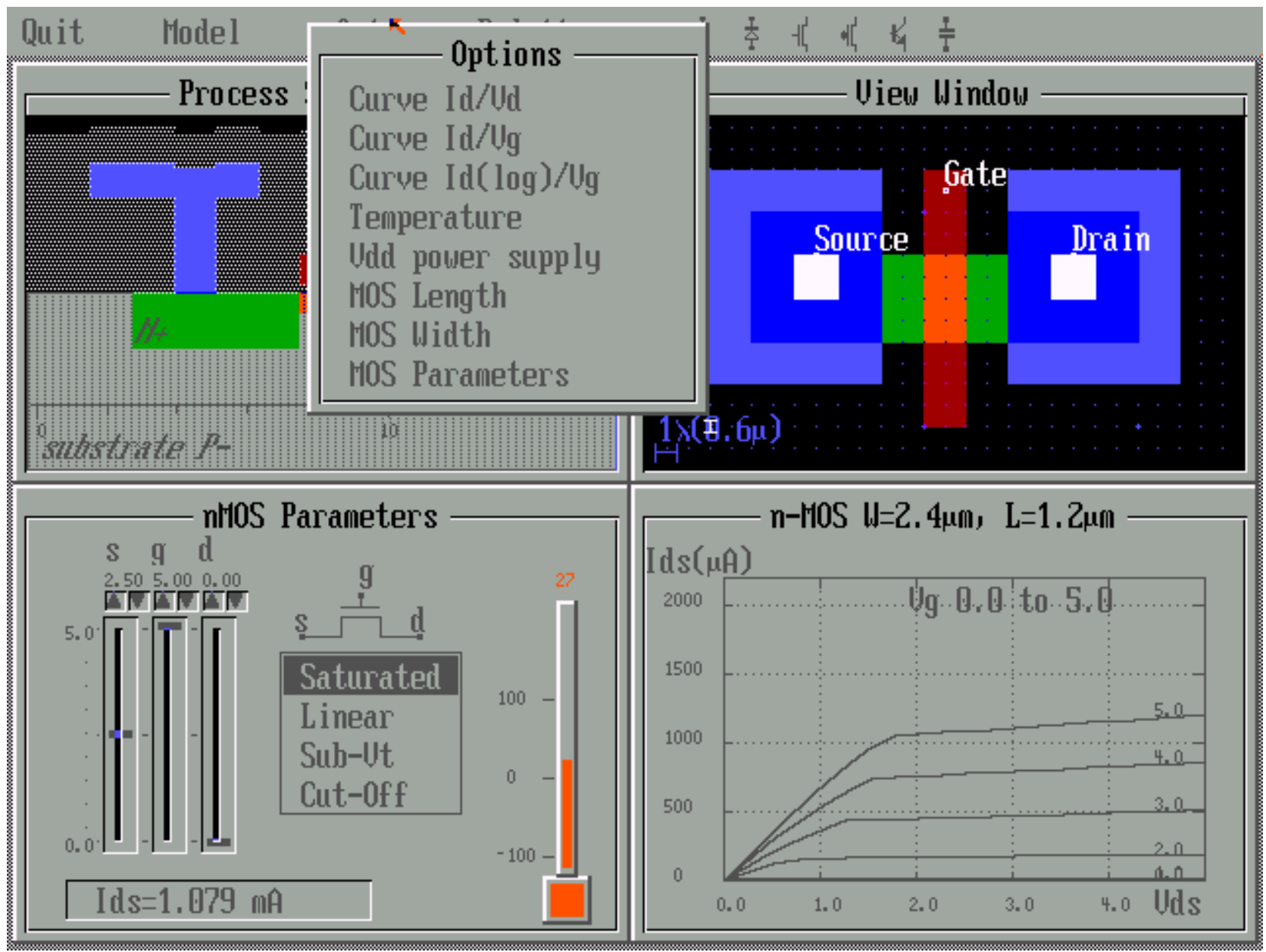

Fig. 13 : Dimensionnement du NMOS.

\subsection{Canal $P$ / canal $N$}

La première comparaison entre les deux types de transistors est au niveau de la réalisation technologique, avec la nécessité d'implanter un puits $N$ (Fig. 14) servant de substrat au transistor à canal $P$.

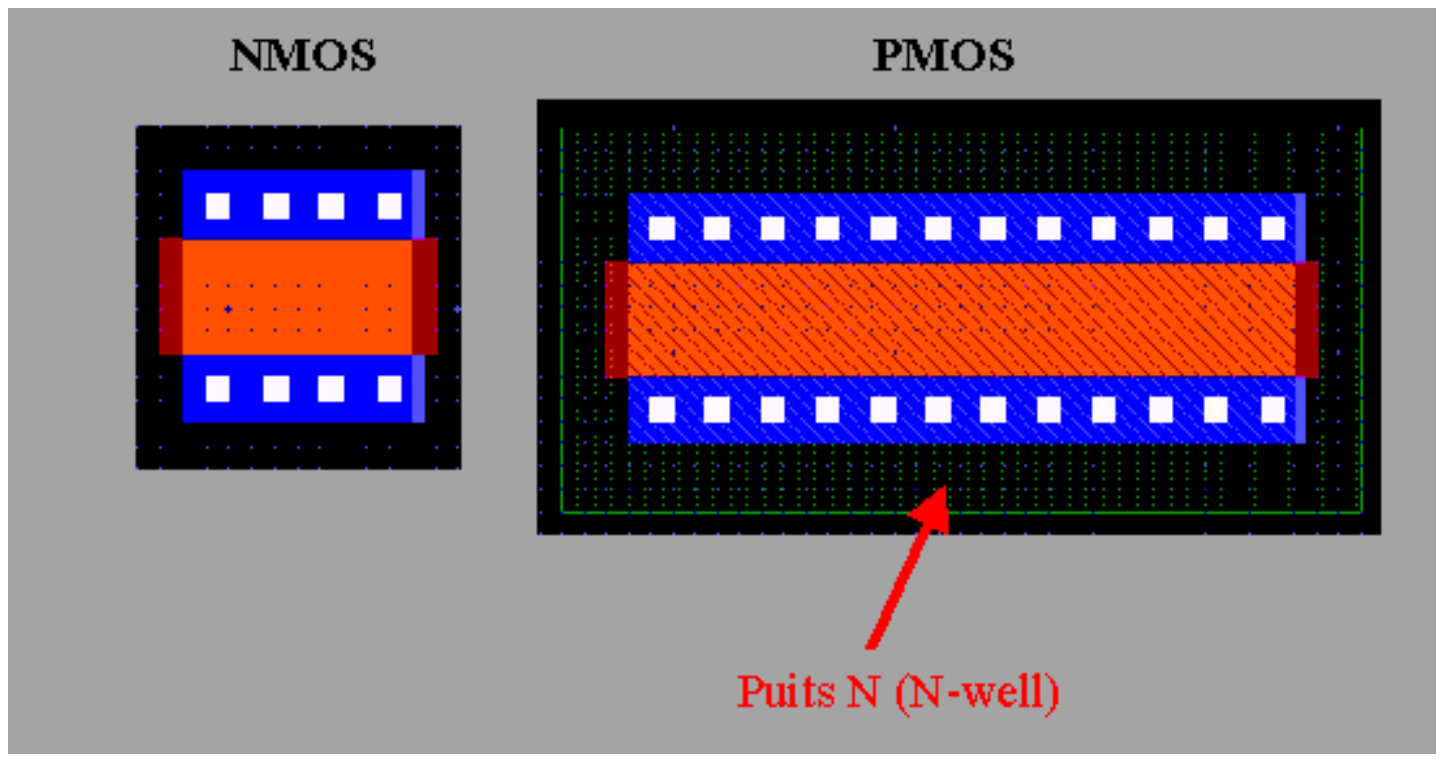

Fig. 14 : Dimensionnement du PMOS/NMOS. 
La seconde comparaison porte sur les caractéristiques électriques des deux types de transistors. À mêmes dimensions et pour des tensions de polarisation et de seuil équivalentes, le courant du PMOS est inférieur à celui du NMOS dans le rapport des mobilités des trous et des électrons.

Pour des applications en logique CMOS, on met ainsi en évidence la nécessité de surdimensionner le transistor à canal $P$ afin de disposer de courants identiques pour un canal $N$ et $P$ :

Ceci est illustré sur la figure 15 .

$$
\frac{W_{\mathrm{p}}}{W_{\mathrm{n}}}=\frac{\mu_{\mathrm{n}}}{\mu_{\mathrm{p}}}
$$

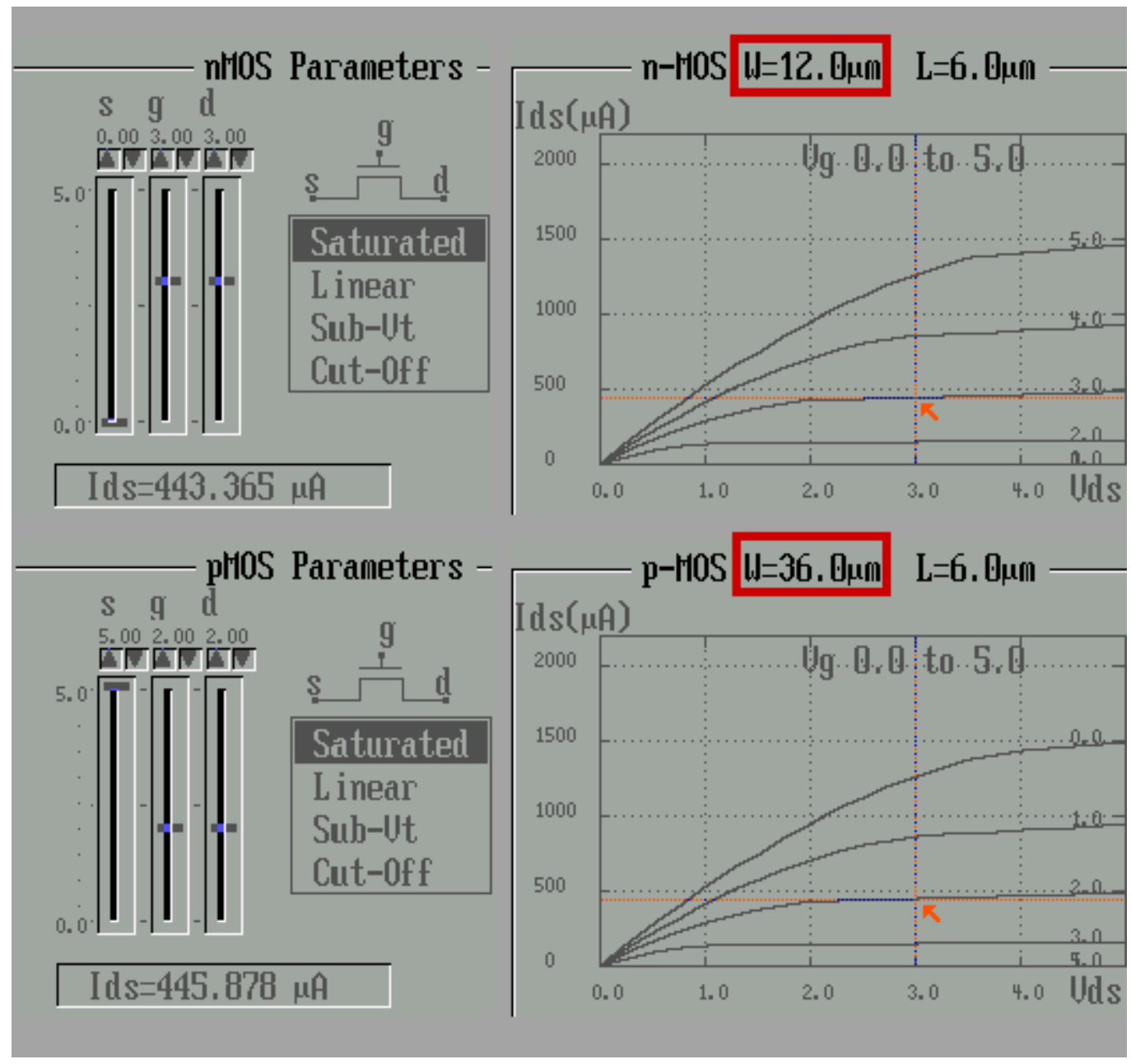

Fig. 15 : Dimensionnement de PMOS et NMOS longs. 


\section{MCARLO}

\subsection{Présentation du logiciel}

Ce module permet de tracer, pour tout point de polarisation de la zone ohmique, les variations de la vitesse moyenne des électrons, de leur énergie potentielle et totale, et de leur température électronique le long du canal d'un transistor à canal $N$. De plus, on peut visualiser la trajectoire d'un électron lors de la traversée du canal. On étudie l'influence de la longueur de la grille $L$ et de la température du réseau $T$ sur ces grandeurs physiques liées aux porteurs.

La réalisation de ce module est basée d'une part sur la résolution des équations de balance et d'autre part sur un simulateur particulaire de type Monte-Carlo très simplifié.

L'écran est divisé en quatre fenêtres (Fig. 16), correspondant à deux types de visualisation, c'est-à-dire :

- la trace d'un porteur par la méthode Monte-Carlo (Fig. 16, en haut à gauche), soit sur la vue en coupe du transistor, soit sur la vue de dessus du canal (Fig. 17);

- les grandeurs physiques données par les équations de balance, soit l'énergie des porteurs (Fig. 16, en haut à droite), leur vitesse (Fig. 16, en bas à gauche), et leur température (Fig. 16, en bas à droite).

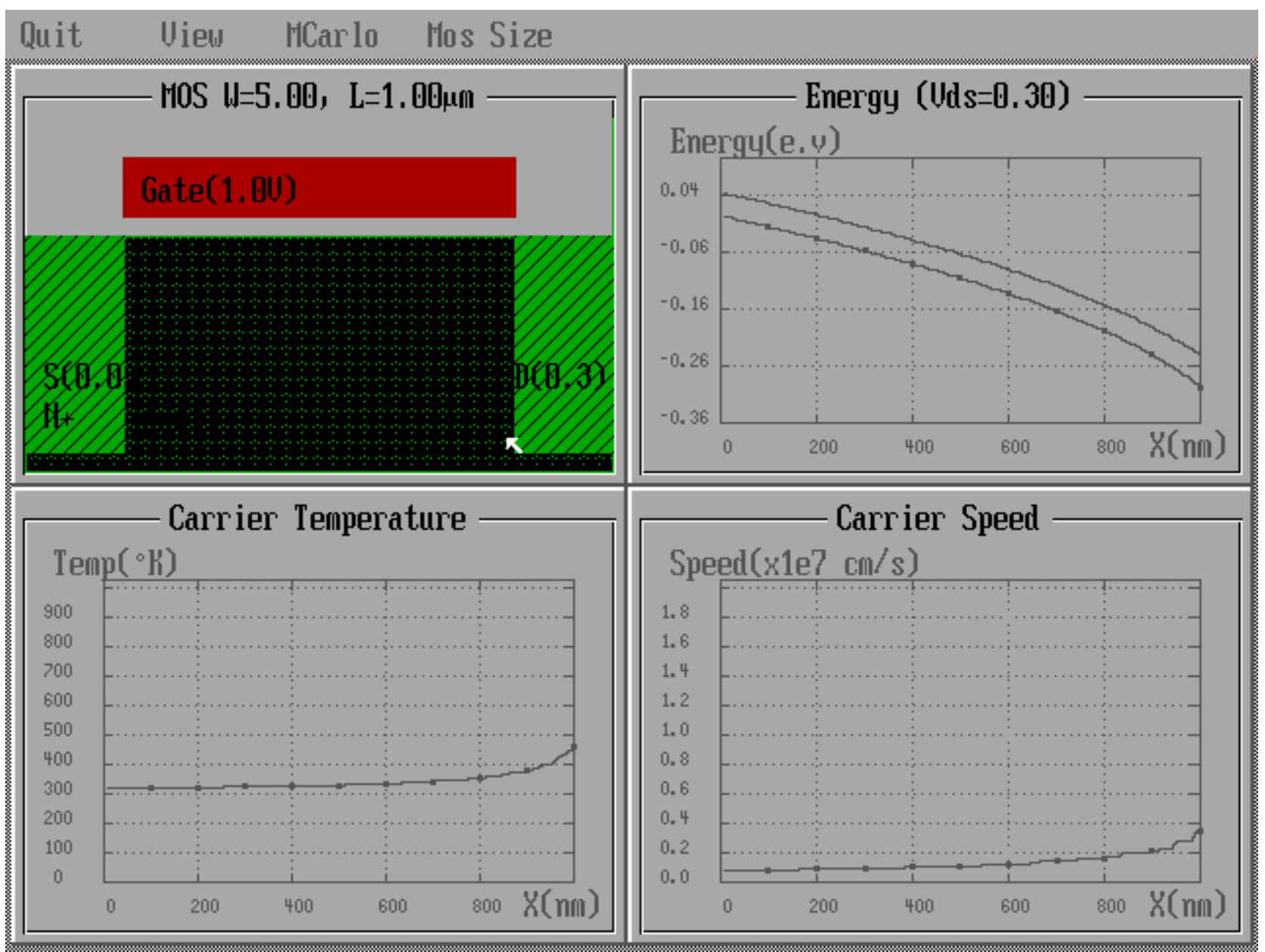

Figure 16. Écran standard de MCARLO. 


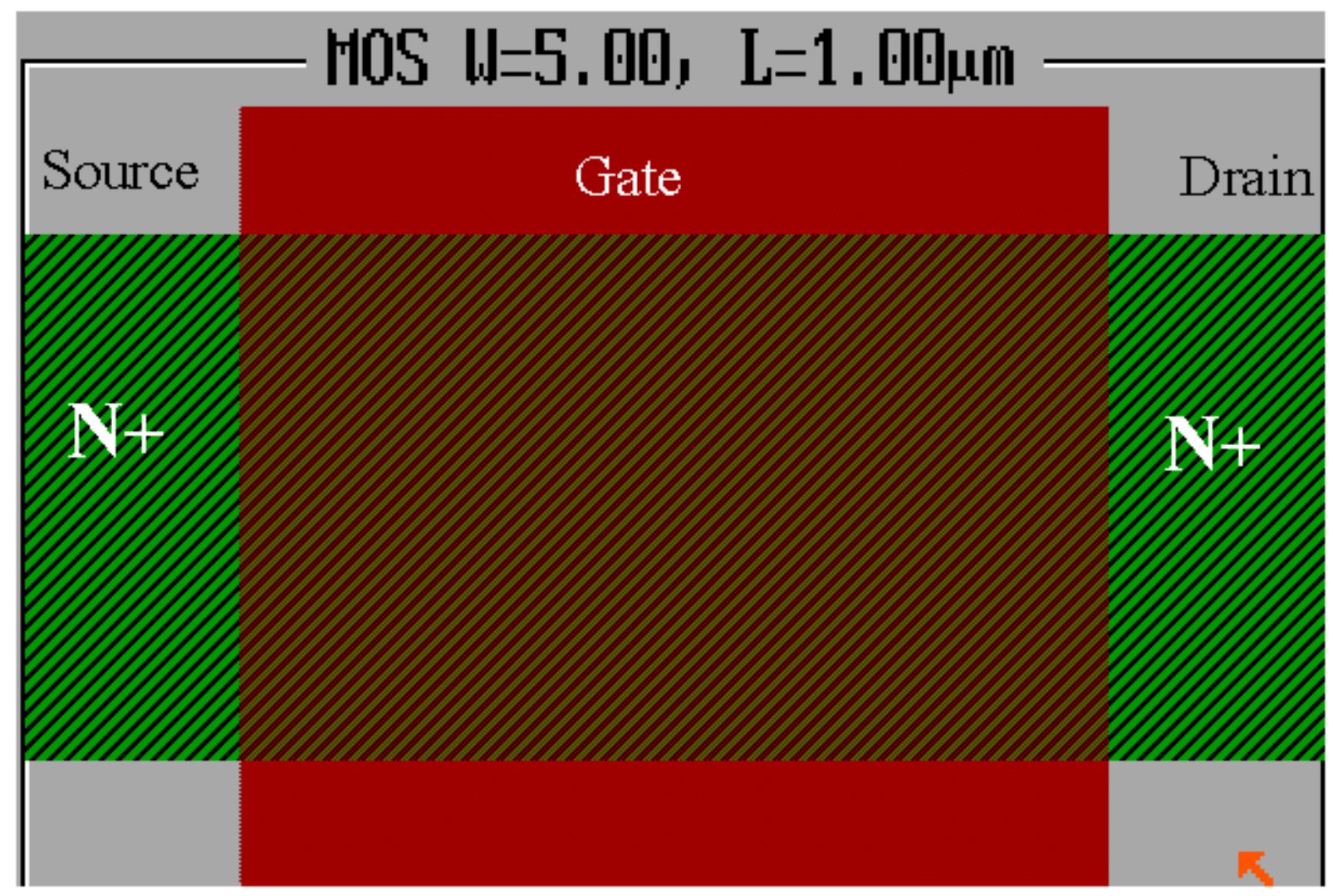

Figure 17. Vue de dessus du canal du transistor.

\section{2. Équations de balance}

Dans un cas unidimensionnel, les équations de balance (ou de relaxation) pour les électrons sont :

- $\quad$ pour la vitesse $v$ :

$$
v \frac{d v}{d x}=-\left[\frac{q E_{x}}{m}+\frac{v}{\tau_{v}}\right]
$$

- pour l'énergie cinétique $^{\varepsilon_{\mathrm{c}}}$ :

$$
v \frac{\mathrm{d} \varepsilon_{\mathrm{c}}}{\mathrm{d} x}=-\left[q E_{\mathrm{z}} u+\frac{\varepsilon_{\mathrm{c}}(\boldsymbol{X})-\varepsilon_{\mathrm{cth}}}{\tau_{\varepsilon}}\right]
$$

où $E_{x}$ est le champ électrique longitudinal, ${ }^{T_{y}}$ et ${ }^{T_{E}}$ sont respectivement les temps de relaxation du moment et de $\varepsilon_{\text {cth }}$. $\varepsilon_{\text {eth }} \equiv 1,5 k_{\mathrm{B}} T \equiv 38 \mathrm{meV}$

l'énergie, ${ }^{\varepsilon_{t h}}$ est l'énergie cinétique thermique donnée par $\varepsilon_{\mathrm{eth}}=1,5 k_{\mathrm{B}} T=38$ mè $T=300 \mathrm{~K}$, où $k_{B}$ est la constante de Boltzmann.

Les temps de relaxation caractérisent le retour à l'état d'équilibre après une faible perturbation. Les mécanismes de relaxation sont les collisions subies par les porteurs.

La résolution de ce système suppose la détermination au préalable des lois de variation : 
- du potentiel électrostatique :

$$
V(x)=\left[\left(V_{G S}-V_{t}\right)=\sqrt{\left(V_{G S}-V_{t}\right)^{2}-\frac{2 I_{D}}{\mu_{n} C_{\text {Gx }} W}}\right]
$$

- du champ électrique longitudinal :

$$
E_{x}(x)=\frac{I_{\mathrm{D}}}{\mu_{\mathrm{m}} C_{\mathrm{sk}} W} \frac{1}{\sqrt{\left(V_{\mathrm{GS}}-V_{\mathrm{t}}\right)^{2}-\frac{2 I_{\mathrm{D}}}{\mu_{\mathrm{n}} C_{\mathrm{ox}}} x}} .
$$

La résolution des équations de balance donne la variation de la vitesse $v$ et de l'énergie cinétique ${ }^{{ }^{\varepsilon_{\mathrm{c}}}}$ et potentielle $\varepsilon_{\mathrm{P}}$ des porteurs le long du canal, soit :

- pour la vitesse :

$$
v(x+\Delta x)=v(x)-\left[\frac{q E_{x}}{m} \frac{1}{v(x)}+\frac{1}{T_{v}}\right] \Delta x
$$

- pour l'énergie cinétique :

$$
\varepsilon_{c}(x+\Delta x)=\varepsilon_{\varepsilon}(x)-\left[q E_{x}+\frac{\varepsilon_{c}(x)-\varepsilon_{c t h}}{T_{\varepsilon}} \frac{1}{v(x)}\right] \Delta x
$$

- et pour l'énergie potentielle :

$$
\mathrm{c}_{p}(x)=-q V(x)
$$

Enfin, la température $T_{e}$ des porteurs est donnée par l'expression ${ }^{\varepsilon_{\mathrm{e}}}=1,5 \mathrm{k}_{\mathrm{B}} T_{\mathrm{e}}$. Ces calculs permettent de tracer les variations le long du canal de la vitesse des porteurs (Fig. 18, en haut), de leur énergie totale et potentielle (Fig. 18, au milieu) et de leur température (Fig. 18, en bas). 


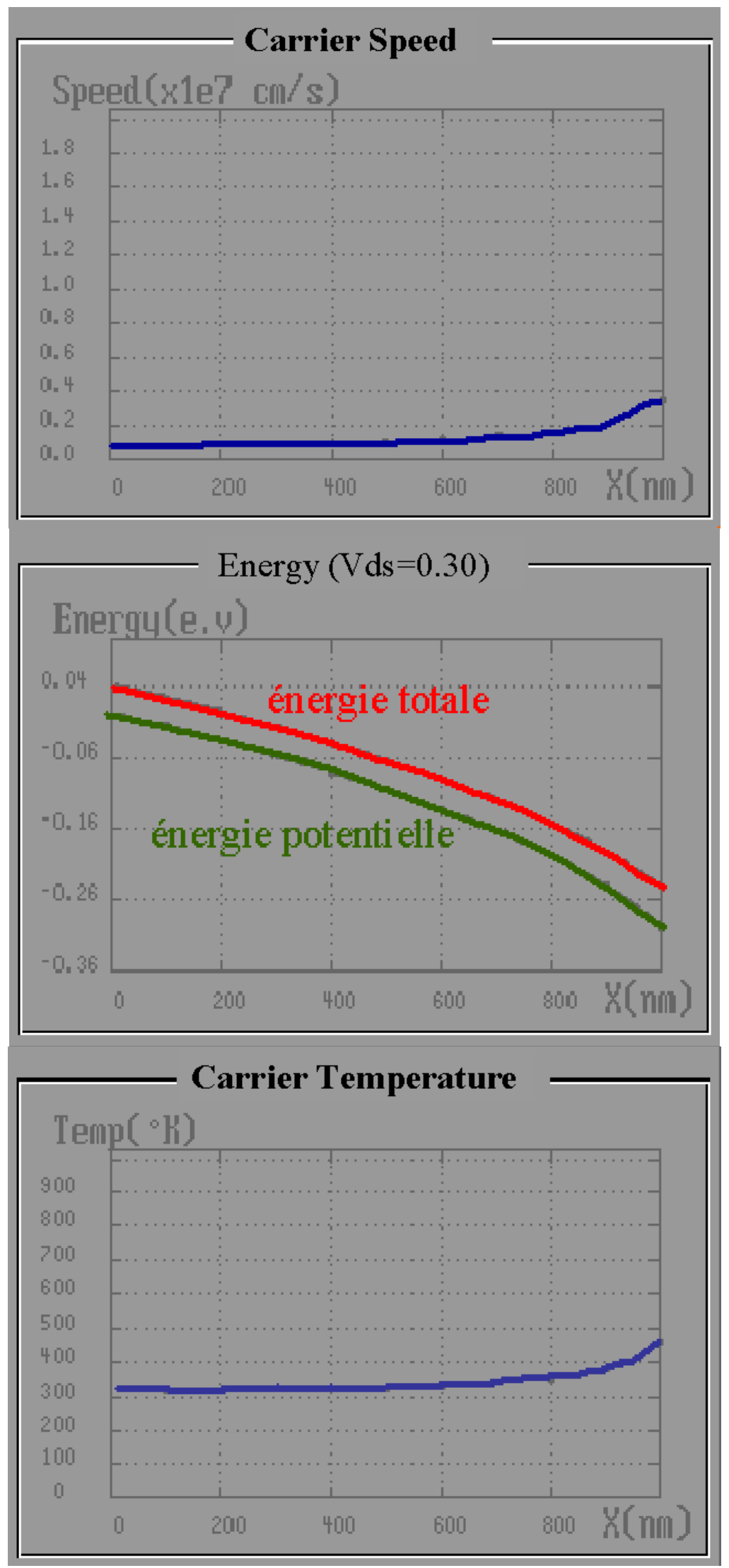

Figure 18. Évolution de la vitesse, des énergies potentielle et totale, de la température des porteurs le long du canal du transistor. (Haut) Vitesse des porteurs. (Milieu) Énergie totale et potentielle des porteurs. (Bas) Température des porteurs. 


\subsection{Mouvement des porteurs par Monte Carlo}

La simulation particulaire Monte Carlo du transport dans un composant à semiconducteur consiste à suivre au cours du temps le comportement individuel de chaque porteur. Le mouvement d'un électron entre deux interactions est un mouvement uniformément accéléré donné suivant $x$ par l'équation :

$$
x(t)=x(0)+t v_{x}(0)-\frac{q t^{2}}{2 m} E_{x}
$$

De plus, de nombreuses collisions perturbent le mouvement de l'électron : interactions avec les vibrations du réseau cristallin, avec les impuretés, électron-électron... Afin de simplifier le traitement, nous ne considérons ici que deux interactions :

- l'une élastique (sans échange d'énergie) et isotrope (distribution isotrope de la vitesse après interaction) de fréquence ${ }^{\lambda_{2}}$ telle que :

$$
\begin{array}{ll}
\text { pour } \varepsilon_{\mathrm{s}}<50 \mathrm{meV} & \lambda_{1}=2 \times 10^{1 \mathrm{~s}} \mathrm{~s}^{-1} \\
\text { pour } \varepsilon_{\mathrm{c}}>50 \mathrm{meV} & \lambda_{1}=1 \times 10^{13} \mathrm{~s}^{-1} .
\end{array}
$$

- l'autre inélastique et isotrope de type émission de phonons de fréquence ${ }^{\lambda_{2}}$ telle que :

$$
\begin{array}{ll}
\text { pour } \varepsilon_{\mathrm{c}}<50 \mathrm{meV} & \lambda_{2}=0 \mathrm{~s}^{-1} \\
\text { pour } \varepsilon>50 \mathrm{meV} & \lambda_{2}=1 \times 10^{1 \mathrm{~s}} \mathrm{~s}^{-1}
\end{array}
$$

Le mouvement est alors décrit comme une suite de vols libres, sous le seul effet du champ électrique, entrecoupés d'interactions (Fig. 19). Les temps de vols libres, la nature des interactions et les directions des vitesses des porteurs après chaque interaction sont tirés au sort. Cette procédure stochastique est qualifiée de Monte Carlo.

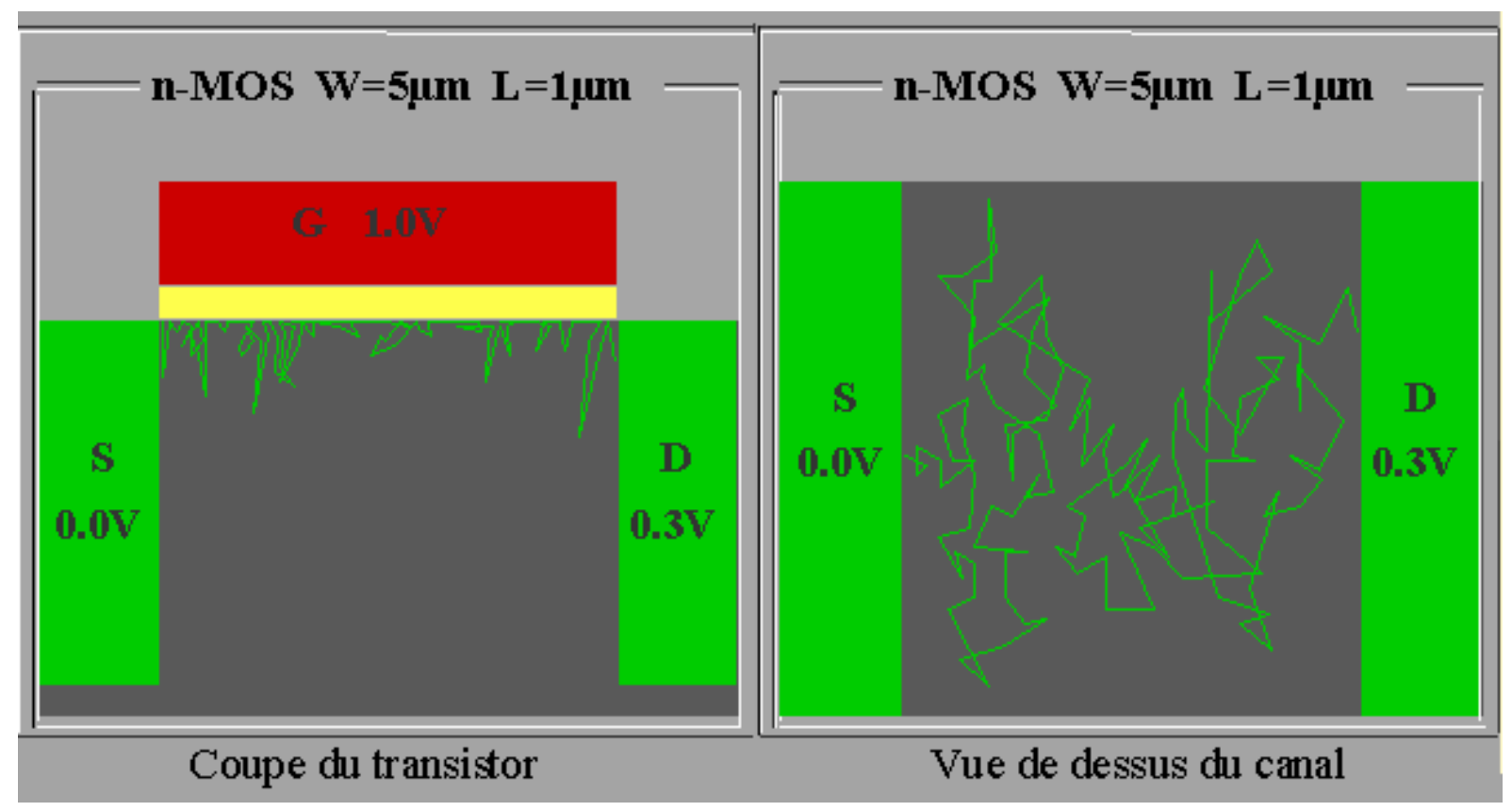

Figure 19. Mouvement des porteurs par méthode de Monte-Carlo. 
Dans le module MCARLO, il est possible de modifier (Fig. 20) :

- la longueur de la grille $L$;

- la largeur de la grille $W$;

- les tensions de polarisation $V_{D S}$ et $V_{G S}$;

- la tension de seuil $V_{t}$;

- l'épaisseur de l'oxyde $T_{o x}$.

On étudie alors l'influence de ces paramètres sur le fonctionnement du transistor.

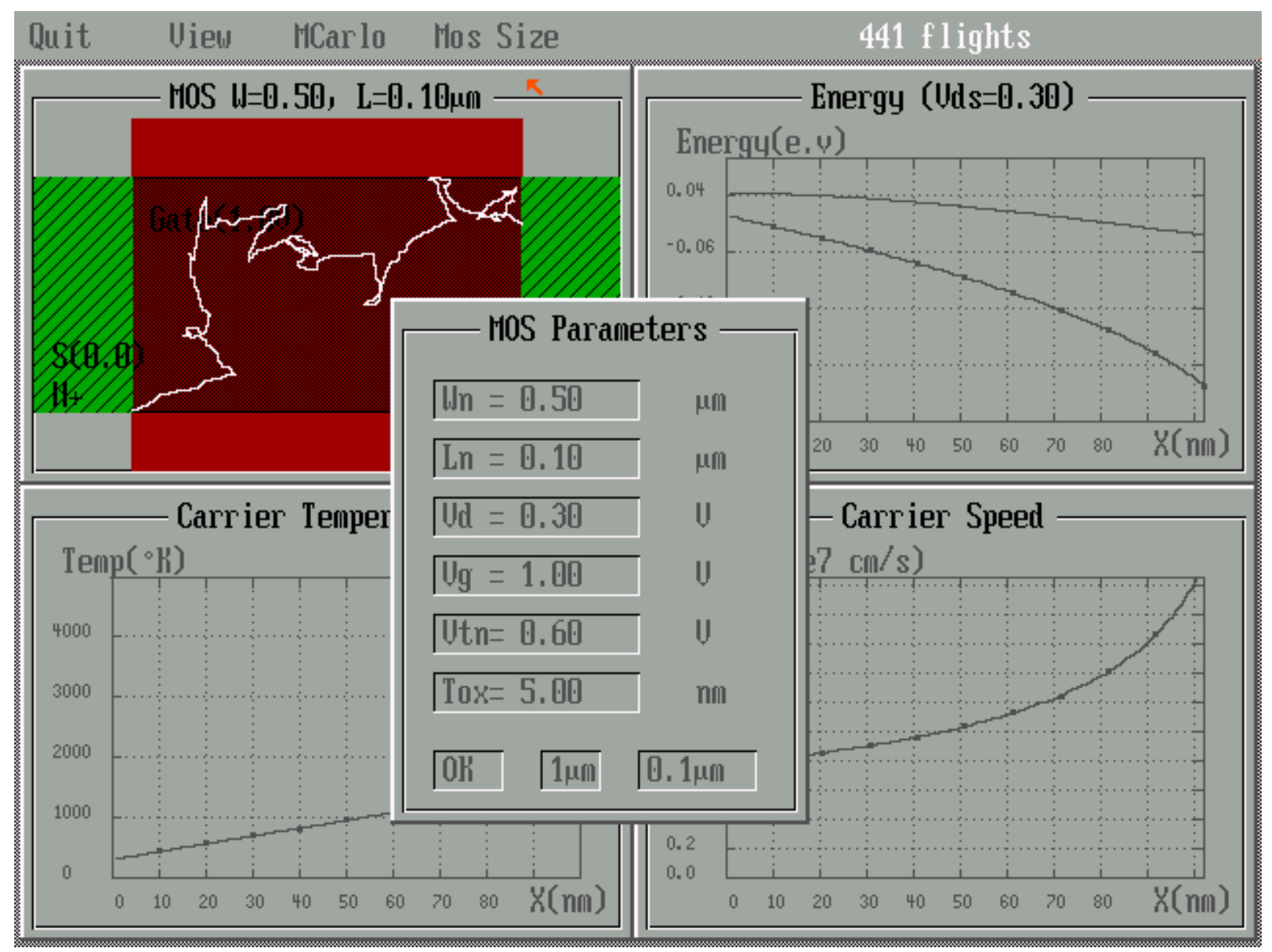

Figure 20. Réglage des paramètres du transistor. 


\subsection{Dimensionnement}

Un exemple d'utilisation de ce module est la mise en évidence du transport non stationnaire, voire balistique, apparaissant avec la réduction de la longueur de la grille. Considérons deux longueurs de grille bien distinctes ( $L=1 \mu \mathrm{m}$ et $0,1 \mu \mathrm{m})$, comme illustré sur la figure 21 .

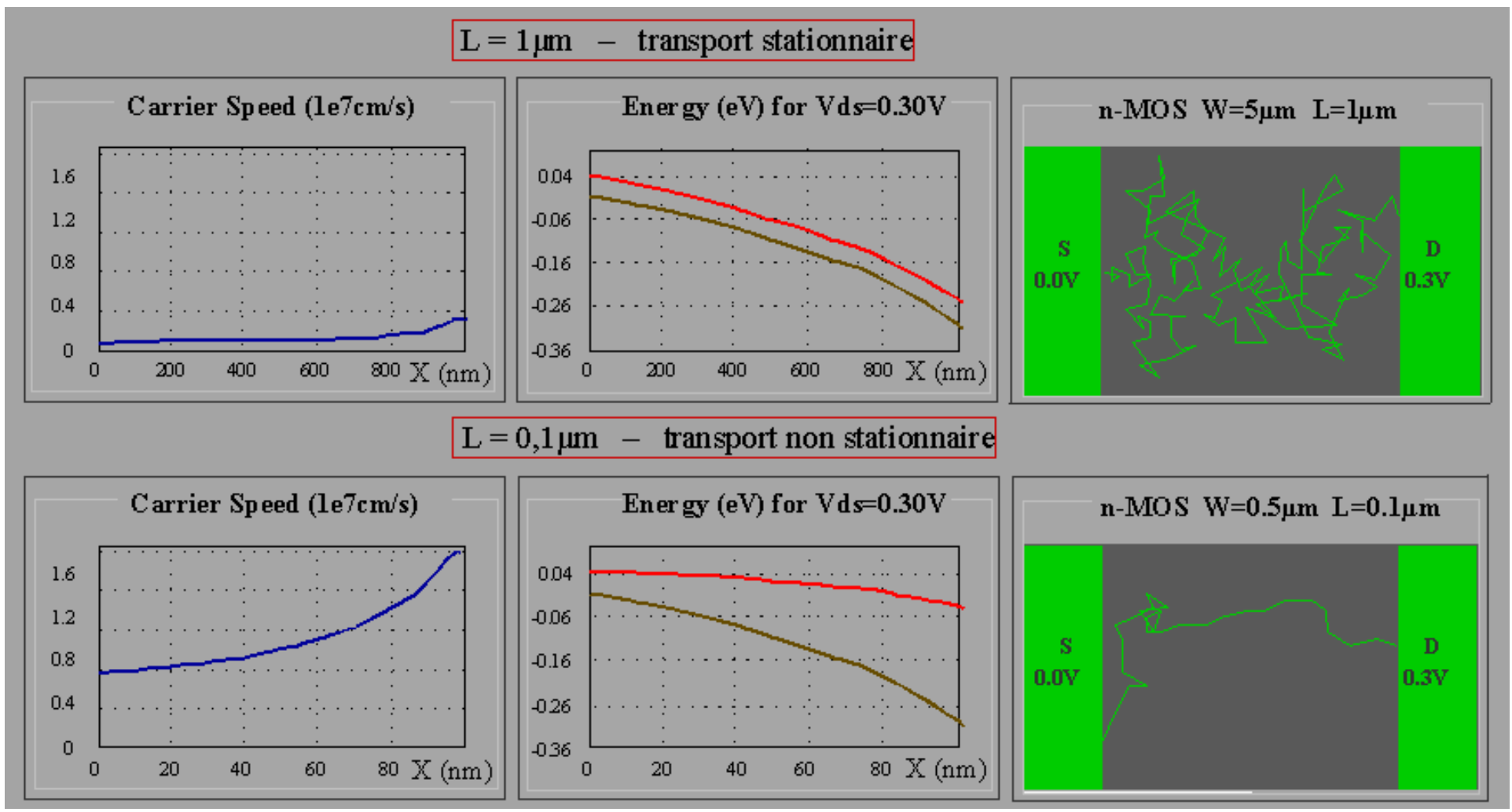

Figure 21. Influence des paramètres géométriques du transistor.

La visualisation de la trajectoire d'un électron montre que plus $L$ est faible et plus le nombre d'interactions que subit un électron dans le canal diminue. Ceci conduit à une augmentation de l'énergie cinétique du porteur comme on peut le voir en comparant les variations d'énergie totale et potentielle ainsi que la vitesse ou encore la température des porteurs. On atteint ainsi des valeurs de la vitesse en bout de canal supérieures à la vitesse stationnaire (phénomène de survitesse) ce qui est favorable à l'amélioration des performances du transistor. 


\section{Conclusion}

Ce TP permet de présenter aux étudiants une approche plus concrète de la physique du composant :

- d'une part, à travers les caractéristiques électriques des transistors qui permettent d'appréhender l'importance d'un dimensionnement correct et le rôle de paramètres physiques tels que la mobilité ou le dopage du substrat, pour un bon fonctionnement en logique CMOS ;

- d'autre part, à travers la variation le long du dispositif de grandeurs physiques telles que les énergies potentielles et totales des porteurs, leur vitesse et leur température. La visualisation des ces grandeurs pour deux longueurs de grille très différentes (très courte et très longue) permet d'introduire l'apparition de nouveaux phénomènes (transport non stationnaire, électrons balistiques) liés à la réduction importante des dimensions.

Enfin, si vous voulez mettre en place ce type de TP, GetMicE (PROF + MCARLO) peut être téléchargé via ftp (ftp://ftp.eea.u-psud.fr/pub/revue-eea/GetMicE.zip).

\section{Remerciements}

Les auteurs remercient Etienne Sicard pour leur avoir permis d'utiliser les logiciels qu'il a développés.

Un grand merci également à Michel Fan pour son aide toujours précieuse et à Philippe Dollfus pour ses commentaires pertinents.

\section{Références bibliographiques}

[1] E. Sicard, Introduction to Microelectronics (INSA Toulouse, 1995).

[2] S. Galdin, E. Sicard, P. Dollfus et F.-X. Musalem, GetMicE: A tutorial of introduction to Micro-Electronics, EAEEIE 96 Oulu (1996).

[3] Silvaco International, Atlas, User's Manual (Santa Clara, USA, 1997).

[4] A. Bournel et S. Galdin-Retailleau, Sensibilisation aux problèmes de la simulation numérique à travers l'étude des composants à semi-conducteurs, CETSIS-EEA'99 Montpellier (Cépaduès-Éditions, 1999),163-166. 